\title{
Controlled finite momentum pairing and spatially varying order parameter in proximitized $\mathrm{HgTe}$ quantum wells
}

\section{Citation}

Hart, Sean, Hechen Ren, Michael Kosowsky, Gilad Ben-Shach, Philipp Leubner, Christoph Brüne, Hartmut Buhmann, Laurens W. Molenkamp, Bertrand I. Halperin, and Amir Yacoby. 2016. "Controlled Finite Momentum Pairing and Spatially Varying Order Parameter in Proximitized HgTe Quantum Wells." Nature Physics 13 (1) (September 19): 87-93. doi:10.1038/nphys3877.

\section{Published Version}

doi:10.1038/nphys3877

\section{Permanent link}

http://nrs.harvard.edu/urn-3:HUL.InstRepos:30805500

\section{Terms of Use}

This article was downloaded from Harvard University's DASH repository, and is made available under the terms and conditions applicable to Other Posted Material, as set forth at http:// nrs.harvard.edu/urn-3:HUL.InstRepos:dash.current.terms-of-use\#LAA

\section{Share Your Story}

The Harvard community has made this article openly available.

Please share how this access benefits you. Submit a story.

Accessibility 


\title{
Controlled Finite Momentum Pairing and Spatially Varying
}

\section{Order Parameter in Proximitized HgTe Quantum Wells}

\author{
Sean Hart ${ }^{\dagger 1}$, Hechen Ren ${ }^{\dagger 1}$, Michael Kosowsky ${ }^{1}$,

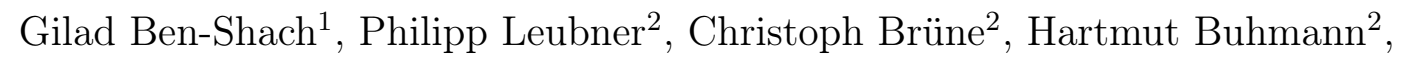 \\ Laurens W. Molenkamp ${ }^{2}$, Bertrand I. Halperin ${ }^{1}$, Amir Yacoby ${ }^{1}$ \\ ${ }^{1}$ Department of Physics, Harvard University, Cambridge, MA, USA \\ ${ }^{2}$ Physikalisches Institut (EP3), Universität Würzburg, 97074 Würzburg, Germany \\ ${ }^{\dagger}$ These authors contributed equally to this work
}

\begin{abstract}
Conventional $s$-wave superconductivity is understood to arise from singlet pairing of electrons with opposite Fermi momenta, forming Cooper pairs whose net momentum is zero [1]. Several recent studies have focused on structures where such conventional $s$-wave superconductors are coupled to systems with an unusual configuration of electronic spin and momentum at the Fermi surface. Under these conditions, the nature of the paired state can be modified and the system may even undergo a topological phase transition [2, 3]. Here we present measurements and theoretical calculations of several $\mathrm{HgTe}$ quantum wells coupled to either aluminum or niobium superconductors and subject to a magnetic field in the plane of the quantum well. By studying the oscillatory response of Josephson interference to the magnitude of the in-plane magnetic field, we find that the induced pairing within the quantum well is spatially varying. Cooper pairs acquire a tunable momentum that grows with magnetic field strength, directly reflecting the response of the spin-dependent Fermi surfaces to the in-plane magnetic field. In addition, in the regime of high electron density, nodes in the induced superconductivity evolve with the electron density in agreement with our model based on the Hamiltonian of Bernevig, Hughes, and Zhang [4]. This agreement allows us to quantitatively extract the value of $\tilde{g} / v_{F}$, where $\tilde{g}$ is the effective g-factor and $v_{F}$ is the Fermi velocity. However, at low density our measurements do not agree with our model in detail. Our new understanding of the interplay between spin physics and superconductivity introduces a way to spatially engineer the order parameter, as well as a general framework within which to investigate electronic spin texture at the Fermi surface of materials.
\end{abstract}


Below a critical temperature and magnetic field, certain materials undergo a phase transition to the superconducting state. Macroscopically identified through effects such as zero resistivity and the Meissner effect [5], superconductors may further be understood microscopically as arising due to pairing of electrons occupying opposite points on the Fermi surface and having opposite spin. Within a conventional setting this interaction results in Cooper pairs with zero net momentum. However, in certain materials the presence of both magnetic order and superconductivity can lead to intrinsically nonzero pairing momentum as the system enters the Fulde-Ferrell-Larkin-Ovchinnikov (FFLO) state [6, 7]. Studies of both $\mathrm{CeCoIn}_{5}$ and $\kappa$-(BEDT-TTF $)_{2} \mathrm{Cu}(\mathrm{NCS})_{2}$ under large external magnetic fields found evidence for coupled magnetic order and superconductivity, although in each material the field strength needed was in excess of $10 \mathrm{~T}$ [8, 9].

Exotic superconductivity has recently come under additional investigation through the goal of combining $s$-wave superconductors with materials whose properties are rarely found among the conventional superconductors. For example, inducing the singlet pairing of an $s$-wave superconductor into a material with strong spin-orbit coupling and reduced dimensionality has been recently considered as a viable platform within which to achieve triplet pairing [10] and topological superconductivity [2, 3], or to engineer a Josephson $\phi_{0}$-junction [11, 12]. Moreover, when a ferromagnetic layer is sandwiched by two superconductors, pairs traversing the junction acquire momentum due to the exchange field within the ferromagnet [13, 14]. Measurements of critical current oscillations in such superconductor-ferromagnetsuperconductor (SFS) junctions have provided evidence for this nonzero pairing momentum [15, 16, 17], although the magnitude of the momentum was effectively untunable due to the typically large exchange fields.

Here we report on coupling between superconducting leads and a two-dimensional electron system realized within $\mathrm{HgTe} / \mathrm{HgCdTe}$ heterostructures in the inverted regime. Due to the interplay between superconductivity, band structure, and the applied magnetic field, we find that the order parameter has an oscillatory component derived from the finite momentum of paired electrons, and that this momentum can be continuously tuned between conventional and unconventional regimes. Our use of only relatively small external magnetic fields $(\leq 4 \mathrm{~T})$ and micron-scale device dimensions introduces a new regime in the exploration of the interplay between superconductivity and spin physics.

To study the effect of magnetic field and band structure on electron pairing, we place two superconducting leads on opposite boundaries of a rectangular section of quantum well. Devices were fabricated at varying angles with respect to the cleavage edges of the crystal (the [110] and [110] axes). The angular alignment corresponds to a rotation angle $\theta$ with respect to the principal crystal axis [100], with $\theta$ defined modulo $\pi / 2$ (Figure 1a, see Supplementary Information I). The width $W$ between the two leads 
is $800 \mathrm{~nm}$ and the length $L$ of the resulting Josephson junction is 4 microns. We study the influence of either niobium or aluminum superconductors by applying a small AC current bias between the two leads while measuring the resultant AC voltage [18]. The aluminum thickness is $15 \mathrm{~nm}$ in order to sustain superconductivity in moderate parallel magnetic fields (Supplementary Information II) [19], while the niobium thickness is $130 \mathrm{~nm}$. Josephson interference is generated by application of small (up to $\sim 10$ $\mathrm{mT}$ ) magnetic fields in the $z$ direction [20]. Throughout, the in-plane coordinate axes are referred to as $x$ and $y$, respectively oriented perpendicular and parallel to the supercurrent flow between leads. The addition of a normal metal topgate allows us to tune the electron density within the junction, thereby providing a means to study superconductivity from the quantum spin Hall regime [21, 22] into the electron-doped regime. In the regime of high electron density and with no parallel magnetic field, junctions show Josephson interference consistent with uniform supercurrent transport through the bulk of the quantum well, shown in Figure $1 \mathrm{~b}$ for a device with aluminum leads.

We primarily study differential resistance with zero applied DC current, due to the efficiency of such measurements in illuminating the structure of the interference pattern. Lower resistance relative to the normal device resistance typically corresponds to elevated critical current (Supplementary Information III). In an aluminum-based junction, in the electron-doped regime and with angle $\theta=\pi / 4$, increasing the magnetic field in the $x$ direction strongly modulates the Josephson interference (Figure 1c). Two distinct regions of decreased resistance are separated by a nodal field of approximately $B_{x}=1.1 \mathrm{~T}$, corresponding to the suppression of induced superconductivity. At each value of the parallel field, we extract the minimum junction resistance as a measure of the strength of superconductivity at that particular field. Plotting these minimum junction resistances highlights the oscillatory effect of parallel field on superconductivity, with the nodal field marked by an arrow (Figure 1d). The suppression of superconductivity at the nodal field directly results from the finite momentum of induced Cooper pairs.

In an aluminum-based junction oriented with $\theta=\pi / 2$, a similar modulation of superconductivity occurs as the parallel field $B_{x}$ grows (Figure 1e, see Supplementary Information IV-V). Although the aluminum leads can sustain superconductivity up to $1.75 \mathrm{~T}$, we only measure this device up to $B_{x}=1$ $\mathrm{T}$ due to constraints on the range of our vector magnet (see Supplementary Information I).

The resistance of a device with niobium leads and $\theta=\pi / 4$ is similarly modulated upon application of a parallel field, with multiple nodes visible as $B_{x}$ increases to $4 \mathrm{~T}$ (Figure 1f). A more detailed measurement highlights the presence of three distinct regions of decreased resistance, separated by bands of high resistance occurring near $B_{x}=0.9 \mathrm{~T}$ and $B_{x}=2.7 \mathrm{~T}$ (Figure 1g). We again extract the minimum junction resistance at each particular parallel field value, demonstrating the oscillatory effect of parallel field on superconductivity (Figure 1h). Nodes of the oscillation, marked by arrows, 
correspond to local maxima in the overall junction resistance.

Despite the differences in fabrication of our devices, the nodal structure is both robust and occurs at nearly the same parallel field magnitudes. These observations suggest that the induced pairing momentum originates in the heterostructures and not the bulk superconductors, and is insensitive to details of the crystal orientation. Since superconductivity arises from pairing of electrons with opposing spins and momenta, it is therefore necessary to examine the nature of both Zeeman coupling and spinorbit coupling within the quantum well.

We model our devices by considering first the quantum well region in the absence of the superconductors, for which a four-band theoretical Hamiltonian $H_{1}$ was proposed as a way to describe the topology of the band structure [4]. We adopt a version of this model to include both the external magnetic field and possible contributions from spin-orbit coupling [23, 24, 25]. The key prediction of the band structure modeling is that the Zeeman coupling from the external field $B_{x}$ modifies the Fermi surfaces in a manner which depends on the nature of the spin-orbit coupling (Supplementary Information VI). As a consequence, the induced superconducting order parameter is expected to oscillate in space, due to a pairing momentum shift with magnitude of order $\hbar \Delta k \approx \tilde{g} \mu_{B} B_{x} / v_{F}$, whose orientation also depends on the spin-orbit coupling. Here $\tilde{g}$ is the in-plane g-factor, and $v_{F}$ is the Fermi velocity.

To theoretically investigate the proximity effect in our quantum wells, we consider a geometry in which the two-dimensional electron gas (2DEG), assumed to have uniform electron density, is contacted by a pair of superconducting leads with a controlled phase difference between them, and we seek to calculate the maximum supercurrent that can be carried between the strips (Figure 3a, see Supplementary Information VII-X for details not presented in the main text). We assume a Hamiltonian $H=H_{1}+H_{2}$, where $H_{2}$ is the coupling between the superconductors and the 2DEG, described by a pairing Hamiltonian of the form

$$
H_{2}=-\int d x d y\left[\Delta(x, y) \Psi^{\dagger}(x, y)+\Delta^{*}(x, y) \Psi(x, y)\right]
$$

Here $\Psi(x, y) \equiv \psi_{\uparrow}(x, y) \psi_{\downarrow}(x, y)$ is an operator which annihilates a singlet pair of electrons in the 2DEG at the point $(x, y)$, while the pair potential $\Delta(x, y)$ is a complex number that depends on the phase of the superconductor and the tunneling amplitude at that point.

We assume that the contacts between the 2DEG and the superconductors occur at the edges of the superconductors, located at $y=0$ and $y=W$, so that we may write

$$
\Delta(x, y)=\lambda_{1}(x) \delta(y)+\lambda_{2}(x) \delta(y-W) \equiv \Delta_{1}(x, y)+\Delta_{2}(x, y)
$$


with $-L / 2<x<L / 2$. We assume that the magnitude of the coupling is constant along each lead, but the phase will vary if there is a perpendicular magnetic field $B_{z} \neq 0$. We choose a gauge where the vector potential points in the $x$ direction, with $A_{x}=-B_{z}(y-W / 2)$, so that the vector potential vanishes along the midline of the $2 \mathrm{DEG}$. If the superconducting strips have identical widths $W_{S C}$, then the couplings $\lambda_{j}$ will have the form

$$
\begin{gathered}
\lambda_{j}(x)=\left|\lambda_{j}\right| e^{2 \pi i \phi_{j}(x)}, \\
\phi_{j}(x)=\phi_{j}(0)+\frac{(-1)^{j-1} x B_{z}\left(W+W_{s c}\right)}{2 \Phi_{0}},
\end{gathered}
$$

with $j=1,2$.

To lowest order in the pairing Hamiltonian $\mathrm{H}_{2}$, the portion of the total energy that depends on the phase difference between the two superconducting leads can be written in the form:

$$
E=-\int d x_{2}\left[\lambda_{2}^{*}\left(x_{2}\right)\left\langle\Psi\left(x_{2}, W\right)\right\rangle_{1}+c . c .\right]
$$

where $\langle\Psi(x, y)\rangle_{1}$ is the order parameter at point $(x, y)$ induced by the superconductor $j=1$. In turn, this may be written in the form

$$
\langle\Psi(x, y)\rangle_{1}=\int d x_{1} \lambda_{1}\left(x_{1}\right) F\left(x, x_{1}, y\right)
$$

where $F$ is the propagator from point $\left(x_{1}, 0\right)$ to point $(x, y)$ for an induced Cooper pair. Depending upon the relative magnitudes of spin-orbit coupling and the Zeeman coupling, the propagator $F$ may take various forms (see Supplementary Information IX). In the limit where either structural inversion asymmetry (SIA) or bulk inversion asymmetry (BIA) is strong compared to the Zeeman coupling, the pair momentum shift orientation is independent of position on the Fermi surface. The shift occurs along an angle $\alpha$ with respect to the $x$ axis, and the propagator is

$$
F\left(x, x_{1}, y\right)=\frac{k_{F}}{8 \pi^{2} v_{F}} \cdot \frac{e^{i \gamma}+e^{-i \gamma}}{\left(x-x_{1}\right)^{2}+y^{2}}, \gamma=\Delta k\left(\sin (\alpha) y+\cos (\alpha)\left(x-x_{1}\right)\right)
$$

When SIA dominates the spin-orbit coupling, a magnetic field $B_{x}$ induces pairing momentum in the $y$ direction, and the order parameter also oscillates in the $y$ direction (Figure $2 \mathrm{a}$ ). When $\Delta k W=\pi / 2$, the first node of the oscillation coincides with the line $y=W$ corresponding to the width of the junction. Increasing the parallel field so that $\Delta k W=3 \pi / 2$ leads to coincidence of the second node and the 
junction width (Figure 2b).

If BIA instead dominates the spin-orbit coupling, when $\theta=0$ the parallel magnetic field induces order parameter oscillations in the $x$ direction (Figure 2c). These oscillations arise due the finite length of the Josephson junction, with amplitudes that are largest near the ends of the mesa. In contrast to the limit of large SIA, with dominant BIA the nodes of the order parameter never coincide with the junction width. Oscillations in the order parameter instead occur with greater frequency along the $x$ direction as the magnetic field increases.

Finally, when the Zeeman coupling dominates the spin-orbit coupling, the pair momentum shift magnitude is isotropic in-plane, but the orientation lies parallel to the direction of Cooper pair propagation. In this limit the propagator is

$$
F\left(x, x_{1}, y\right)=\frac{k_{F}}{8 \pi^{2} v_{F}} \cdot \frac{e^{i \gamma}+e^{-i \gamma}}{\left(x-x_{1}\right)^{2}+y^{2}}, \gamma=\Delta k \sqrt{\left(x-x_{1}\right)^{2}+y^{2}}
$$

Here the induced order parameter oscillates along both the $x$ and $y$ directions (Figure 2d). Although the shape of the order parameter resembles the limit of strong SIA, the possibility to oscillate in all in-plane directions prevents a node from forming along a line of constant $y$.

We can link the order parameter oscillations to the Josephson energy $E$ by integrating over the second superconducting lead at position $y=W$, as in equation (5). By then differentiating with respect to the phase difference $\phi_{2}(0)-\phi_{1}(0)$ we find the current-phase relation of the junction, which is then maximized with respect to the phase difference to obtain the critical current.

When SIA dominates the spin-orbit coupling, the critical current periodically disappears when the nodal condition $\Delta k W=(2 n+1) \pi / 2$ is satisfied (Figure $3 \mathrm{~b}$ ). These nodal points in the critical current correspond to spatial nodes of the induced order parameter coinciding with the positions of the leads, so that the supercurrent is completely suppressed. Microscopically, these oscillations of the order parameter correspond to finite momentum pairing of electrons, as diagrammed in the inset of Figure $3 \mathrm{~b}$. In the limit of strong SIA, the Fermi surfaces oppositely shift in the $y$ direction, so that Cooper pairs form internally to each surface with finite wavevector $\Delta k \hat{y}$.

The predicted interference with strong SIA resembles the nodal pattern we observe experimentally. However, in niobium-based devices we also observe that superconductivity weakens and that nodes occur over an increasingly large range of parallel field strengths as the parallel field increases. To model this behavior, we consider the effect of structural imperfections in the interface between the superconducting leads and the quantum well. These would introduce random fluctuations in the direction of the inplane magnetic field at each interface, leading to a random component of the pairing momentum in 
the $x$ direction that grows linearly with the in-plane field. Hence, we introduce a random phase $\chi \propto$ $\left(R_{1}\left(x_{1}\right)-R_{2}\left(x_{2}\right)\right) B_{x}$, where the random variables $R_{1}\left(x_{1}\right)$ and $R_{2}\left(x_{2}\right)$ correspond to fluctuations in the direction of the parallel field at each interface (see Supplement XI for details). With this randomness, the calculated critical currents diminish in magnitude as the in-plane field increases, and nodes occur over a larger range of in-plane field, in agreement with our experimental observation (Figure 3c).

Considering, instead, BIA as the dominant source of spin-orbit coupling, when the junction is aligned to the [100] or [010] crystal direction, the order parameter oscillates in the $x$ direction. This oscillation corresponds to shifting of the Fermi surfaces oppositely along $x$, so that Cooper pairs form internally to each surface with wavevector $\Delta k \hat{x}$ (Figure 3d). Since the real-space supercurrent density and the Josephson critical current can be regarded as Fourier conjugates [26], this pairing momentum results in finite weight of the interference at a particular magnitude of $B_{z}$ that grows linearly with the parallel field, forming a ' $\mathrm{V}$ ' shape. In our measurements of the device oriented with $\theta=\pi / 2$, this splitting would be seen in the limit of strong BIA, but is not observed experimentally (Figure 1e, Supplementary Information V). Additionally, when junctions are fabricated at an angle $\theta=\pi / 4$, with strong BIA the behavior is expected to shift from that shown in Figure 3d to the nodal structure in Figure 3b. Since we instead observe behavior that does not depend on the crystal orientation, we conclude that BIA in our heterostructure is relatively weak. This conclusion agrees with a previous measurement of Shubnikov-de Haas oscillations in a HgTe quantum well, which was found to be consistent with strong SIA and weak BIA [27].

In the limit of overall weak spin-orbit coupling, the order parameter oscillates in both in-plane directions. Zeeman coupling at finite values of $B_{x}$ leads to two concentric Fermi surfaces with opposite spin polarization, so that pairing occurs between surfaces with momentum in all in-plane directions (Figure 3e). Increasing the parallel magnetic field causes the interference to both spread in $B_{z}$ and periodically oscillate, a hybrid of the two above cases. Characteristically, at each node the two interference fringes adjacent to the central fringe combine to form the subsequent central fringe, a direct result of the inability to form nodes in the order parameter along lines of constant $y$. Although it is possible that this behavior is present in the device with niobium leads, the nodal pattern is more consistent with strong SIA with aluminum leads at high density. In the limit of overall weak spin-orbit coupling, with the random phase $\chi$ the modeled interference successfully reproduces many aspects of the behavior observed in the niobium device, but is still inconsistent with the aluminum devices (Figure 3f).

As an additional study into the nature of electron pairing momentum, we explore the evolution of the minimum junction resistance at different parallel field $B_{x}$ values, while energizing the global topgate to modify the bulk electron density. Devices used for these measurements were aligned such that $\theta=\pi / 4$, 
corresponding to the devices of Figure 1c,d,f-h. At the most positive gate voltage, as the magnetic field is increased the niobium device displays the narrow node of increased resistance near $B_{x}=0.9 \mathrm{~T}$ (Figure 4a). As before, an additional wider node is present near $B_{x}=2.7 \mathrm{~T}$. When the top gate voltage is lowered to $-5 \mathrm{~V}$, the field magnitude of the lower node increases, first slowly and then more rapidly. In the device with aluminum leads, a similar nodal structure is observed, with the magnitude of the node weakly increasing as the top gate voltage is lowered from $0.5 \mathrm{~V}$ to $0 \mathrm{~V}$ (Figure $4 \mathrm{~b}$ ).

The dependence of the node magnitude on density can be calculated within the framework of our model based on BHZ theory, here assuming the presence of SIA due to a perpendicular electric field equal to $10 \mathrm{mV} / \mathrm{nm}$ (Supplementary Information VI). Since the magnitude of the induced Cooper pair momentum is approximately $\hbar \Delta k \approx \tilde{g} \mu_{B} B_{x} / v_{F}$, the dependence of both $\tilde{g}$ and $v_{F}$ on the electron density will directly influence the magnitude of the parallel field needed to satisfy the nodal condition $\Delta k W=(2 n+1) \pi / 2$. Due to the inverted nature of the bands, the g-factors in the conduction bands are expected to evolve from -20.5 toward zero as the Fermi wavevector decreases [25], while the expected magnitudes of the Fermi velocities first decrease slightly and then more rapidly fall to zero (Figure 4c, d). With these considerations we expect the magnitudes of the induced pairing wavevectors at $1 \mathrm{~T}$ to fall to zero from values near $1.2 / \mu \mathrm{m}$ as the Fermi wavevector decreases (Figure 4e). As a result, the magnetic field needed to satisfy the nodal condition increases as the electron density decreases, finally diverging at zero electron density (calculated in black dotted lines in Figure 4a-b). Although the overall evolution agrees well with the expectation from BHZ theory, we find that our measurements on niobium and aluminum devices respectively yield values of $\tilde{g} / v_{F}$ that are approximately 1.9 and 1.4 times greater than those expected theoretically (see Supplement VI).

Several aspects of the density-dependent data do not fall into the modeling framework discussed above, and are interesting for further consideration. First, we expect that the position of the node associated with induced Cooper pair momentum should occur at higher parallel magnetic field as the density is reduced, a behavior that we observe only at high density. As the density is further reduced, the magnitude of the nodal field eventually begins to decrease, an element of our model that is not present and remains to be understood, but could possibly be explained by a finite g-factor at zero density. Second, in the aluminum device, the region of reduced resistance occuring above the first node appears to be strongest near top gate voltages equal to $-0.9 \mathrm{~V}$ and $0.5 \mathrm{~V}$. We observe that these two regions of reduced resistance are connected by a region in which the resistance is more weakly reduced, but we have no reason to expect that the reduction in resistance above the first node should vary as the density decreases.

Our measurements demonstrate that a parallel magnetic field can be used both to tune the momen- 
tum of Cooper pairs in a material and to clarify the nature of spin-orbit coupling in that material. A major current goal of condensed matter physics is to understand the nature of the superconductivity that results when electron pairing is combined with materials possessing exotic spin textures. Therefore our new understanding that the superconducting order parameter can be engineered in space may be utilized to investigate spin physics within a broad range of materials including InAs-based quantum wells or the surfaces of three-dimensional topological insulators. Our method to tune the Josephson energy could find additional application in the field of quantum information processing, where direct control of the energy levels in a single superconducting qubit could provide a powerful tool for the investigation and optimization of qubit coherence.

\section{References}

[1] Bardeen, J., Cooper, L. N. \& Schrieffer, J. R. Microscopic Theory of Superconductivity. Phys. Rev. 108, 1175 (1957).

[2] Fu, L. \& Kane, C. L. Superconducting Proximity Effect and Majorana Fermions at the Surface of a Topological Insulator. Physical Review Letters 100, 096407 (2008).

[3] Sau, J. D., Lutchyn, R. M., Tewari, S. \& Sarma, S. D. Generic new platform for topological quantum computation using semiconductor heterostructures. Physical Review Letters 104, 040502 (2010).

[4] Bernevig, B. A., Hughes, T. L. \& Zhang, S.-C. Quantum Spin Hall Effect and Topological Phase Transition in HgTe Quantum Wells. Science 314, 1757-1761 (2006).

[5] Meissner, W. \& Ochsenfeld, R. Ein neuer Effekt bei Eintritt der Supraleitfahigkeit. Naturwissenschaften 21 (44), 787-788 (1933).

[6] Fulde, P. \& Ferrell, R. A. Superconductivity in a strong spin-exchange field. Phys. Rev. 135, A550-A564 (1964).

[7] Larkin, A. I. \& Ovchinnikov, Y. N. Inhomogeneous state of superconductors. Sov. Phys. JETP 20, 762-769 (1965).

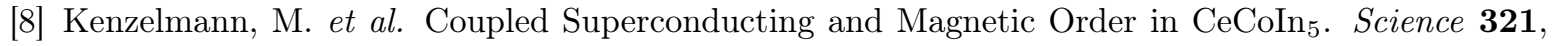
1652-1654 (2008). 
[9] Mayaffre, H. et al. Evidence of Andreev bound states as a hallmark of the FFLO phase in $\kappa$ (BEDT-TTF $)_{2} \mathrm{Cu}(\mathrm{NCS})_{2}$. Nature Physics 10, 928-932 (2014).

[10] Reeg, C. R. \& Maslov, D. L. Proximity-induced triplet superconductivity in Rashba materials. arXiv:1508.03623v1 (2015).

[11] Yokoyama, T., Eto, M. \& Nazarov, Y. V. Anomalous Josephson effect induced by spin-orbit interaction and Zeeman effect in semiconductor nanowires. Physical Review B 89, 195407 (2014).

[12] Dolcini, F., Houzet, M. \& Meyer, J. S. Topological Josephson $\phi_{0}$ junctions. Physical Review B 92, $035428(2015)$.

[13] Buzdin, A. I., Bulaevskii, L. N. \& Panyukov, S. V. Critical-current oscillations as a function of the exchange field and thickness of the ferromagnetic metal (F) in an S-F-S josephson junction. Pis'ma Zh. Eksp. Teor. Fiz. 35, 147-148 (1982).

[14] Demler, E. A., Arnold, G. B. \& Beasley, M. R. Superconducting proximity effects in magnetic metals. Physical Review B 55 (1997).

[15] Kontos, T. et al. Josephson Junction through a Thin Ferromagnetic Layer: Negative Coupling. Physical Review Letters 89 (2002).

[16] Sellier, H. et al. Temperature-induced crossover between 0 and pi states in S/F/S junctions. Physical Review B 68 (2003). 054531.

[17] Frolov, S. M. et al. Measurement of the current-phase relation of superconductor/ferromagnet/superconductor pi Josephson junctions. Physical Review B 70 (2004). 144505.

[18] Oostinga, J. B. et al. Josephson Supercurrent through the Topological Surface States of Strained Bulk HgTe. Phys. Rev. X 3, 021007 (2013).

[19] Meservey, R. \& Tedrow, P. M. Properties of Very Thin Aluminum Films. Journal of Applied Physics 42, 51 (1971).

[20] Tinkham, M. Introduction to Superconductivity (Dover Publications, Inc., 2004).

[21] Konig, M. et al. Quantum Spin Hall Insulator State in HgTe Quantum Wells. Science 318, 766-770 (2007).

[22] Hart, S. et al. Induced Superconductivity in the Quantum Spin Hall Edge. Nature Physics 10, 638-643 (2014). 
[23] Rothe, D. G. et al. Fingerprint of different spin-orbit terms for spin transport in HgTe quantum wells. New Journal of Physics 12, 065012 (22pp) (2010).

[24] Weithofer, L. \& Recher, P. Chiral Majorana edge states in HgTe quantum wells. New Journal of Physics 15, 085008 (2013).

[25] Konig, M. et al. The Quantum Spin Hall Effect: Theory and Experiment. Journal of the Physical Society of Japan 77, No. 3, 031007 (2008).

[26] Dynes, R. C. \& Fulton, T. A. Supercurrent Density Distribution in Josephson Junctions. Physical Review B: Condensed Matter and Materials Physics 3, Number 9, 3015-3023 (1971).

[27] Gui, Y. S. et al. Giant spin-orbit splitting in a HgTe quantum well. Physical Review B 70, 115328 (2004).

Acknowledgments: We acknowledge Ewelina M. Hankiewicz and Grigory Tkachov for theoretical discussions. This work was supported by the NSF DMR-1206016, by the STC Center for Integrated Quantum Materials under NSF Grant No. DMR-1231319 and by Microsoft Corporation Project Q. We acknowledge additional financial support from the German Research Foundation (The Leibniz Program, Sonderforschungsbereich 1170 "Tocotronics" and Schwerpunktprogramm 1666), the EU ERC-AG program (Project 3-TOP) and the Elitenetzwerk Bayern IDK "Topologische Isolatoren".

Author Contributions: The experiment is a collaboration between the Harvard and Würzburg experimental groups. S.H., H.R., M.K., G. B.-S., B. I. H., and A.Y. carried out the theoretical modeling and analysis.

Author Information: The authors declare no competing financial interests. Correspondence and requests for materials should be addressed to yacoby@physics.harvard.edu. 

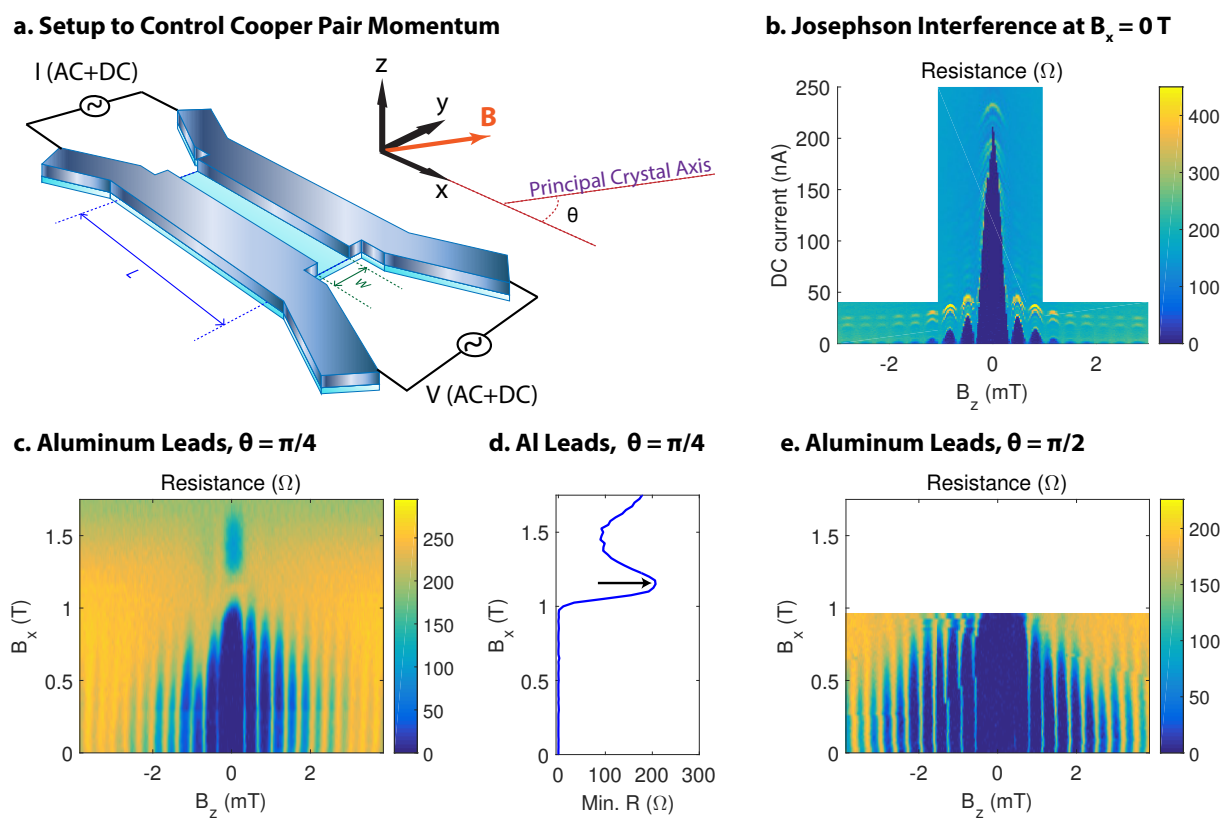

d. Al Leads, $\theta=\pi / 4$
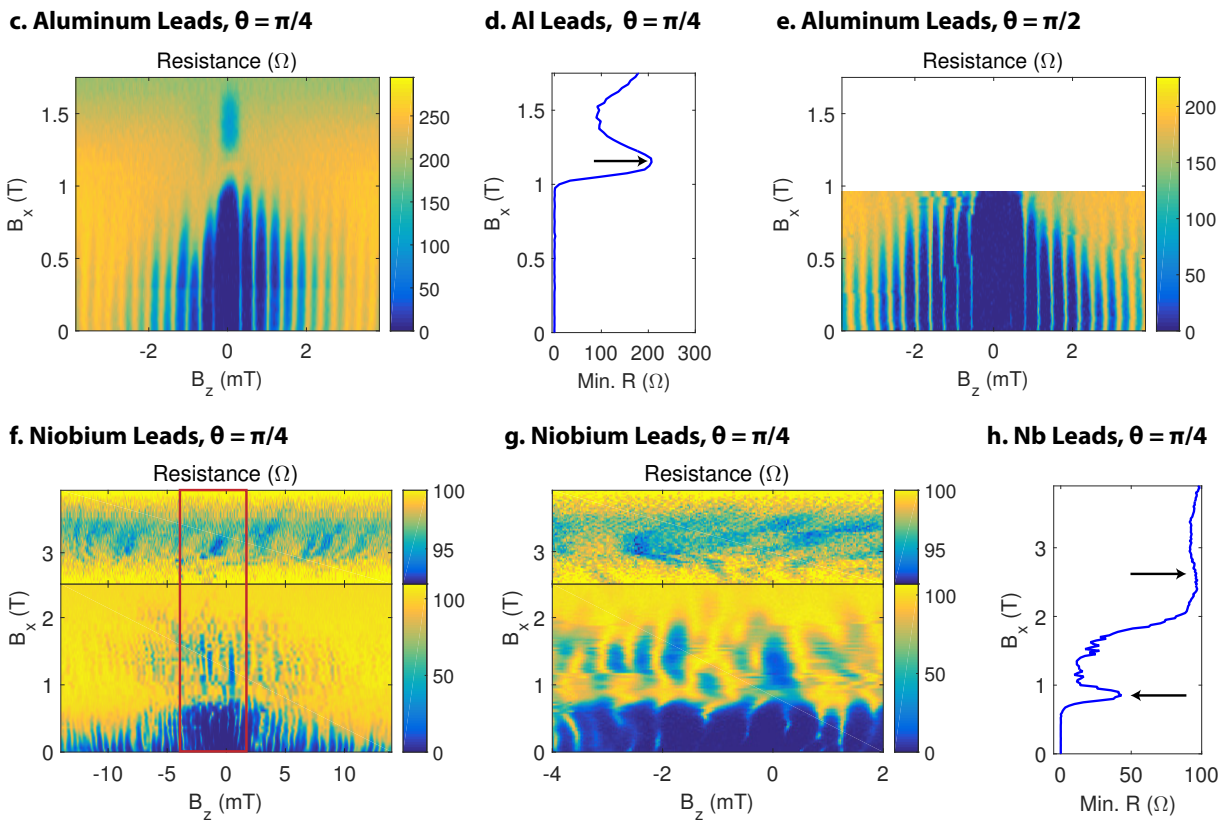

h. Nb Leads, $\theta=\pi / 4$

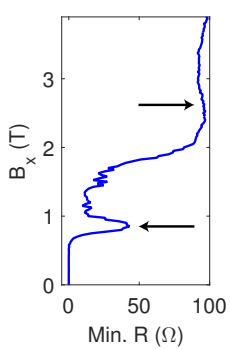

Figure 1: Experimental control of the order parameter and of pairing momentum. (a) Two superconducting leads, composed of either aluminum or niobium, couple to a rectangular section of $\mathrm{HgTe}$ quantum well to form a Josephson junction. The width $W$ separating the leads is always $800 \mathrm{~nm}$, while the length $L$ of the junction is always 4 microns. The resistance of the junction is monitored by applying a small $\mathrm{AC}$ current bias (typically $\sim 1 \mathrm{nA}$ ) and concurrently measuring the resulting $\mathrm{AC}$ voltage. Further sourcing DC current allows measurement of critical currents and normal device resistance. The external magnetic field $\vec{B}$ contains a small $z$ component to generate Josephson interference, while here the larger $x$ component couples significantly to the spin-degree of freedom. Junctions may be oriented at an angle $\theta$ with respect to the [100] principal crystal axis, modulo $\pi / 2$. (b) In the electron-doped regime, the devices show Josephson interference consistent with transport through a doped bulk. In all subsequent measurements, zero DC current is applied. (c) The differential resistance of a junction with aluminum leads oscillates due to Josephson interference as the perpendicular field varies. Increasing the parallel field modulates the strength of induced superconductivity. (d) Plotting the minimum resistance at each value of $B_{x}$ demonstrates the presence of a nodal resistance maximum near $B_{x}=1.1 \mathrm{~T}$. (e) In an aluminum-based device oriented with $\theta=\pi / 2$, increasing the parallel field similarly modifies the resistance. (f) In a junction with niobium leads, a similar modulation of the resistance occurs. (g) A more detailed study of the space outlined in red in (f) highlights three regions of decreased resistance separated by bands of high resistance near $B_{x}=0.9 \mathrm{~T}$ and $B_{x}=2.7 \mathrm{~T}$. In both (f) and $(\mathrm{g})$, the decreased resistance above $2.5 \mathrm{~T}$ is highlighted via a stretched color scale. (h) The minimum resistance at each value of $B_{x}$ further shows the oscillatory nature of the superconductivity as the parallel field increases. Successively higher nodes (marked by arrows) occupy broader regions of parallel field, while superconductivity also weakens as the parallel field increases. 


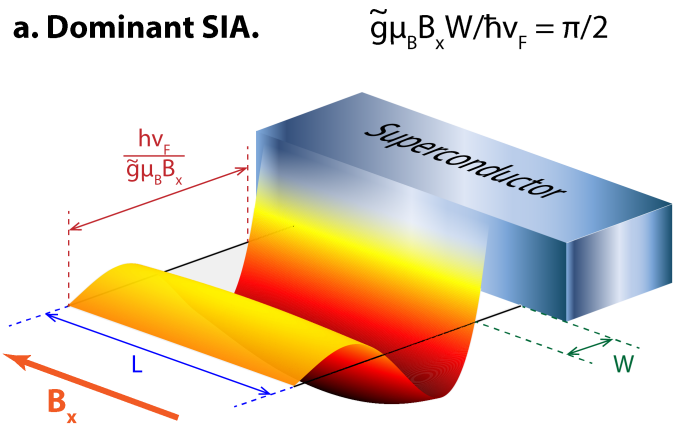

b. Dominant SIA. $\quad \tilde{g} \mu_{B} B \mathrm{~B} / \hbar v_{F}=3 \pi / 2$
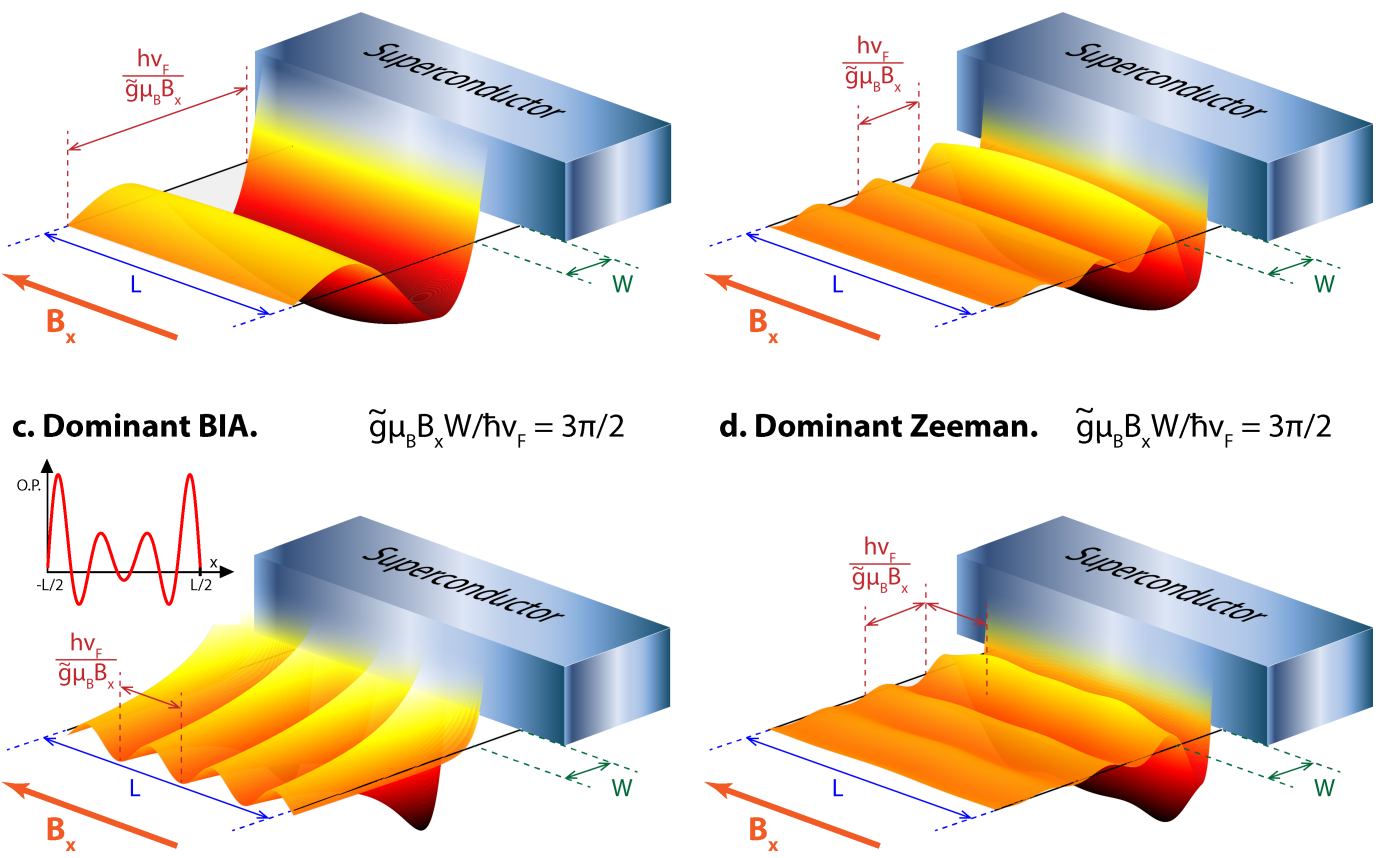

d. Dominant Zeeman. $\quad \tilde{g} \mu_{B} B \mathrm{~B} / \hbar v_{F}=3 \pi / 2$

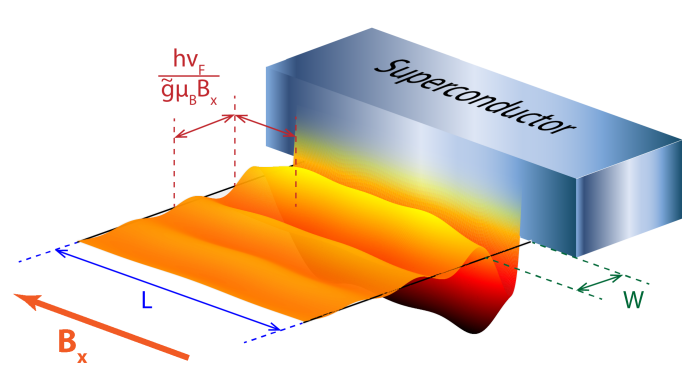

Figure 2: Theoretical prediction for the spatially varying order parameter near a single superconducting lead, with $B_{z}=0$. (a) With dominant SIA, application of an in-plane magnetic field $B_{x}$ induces oscillations of the order parameter in the $y$ direction, with wavelength $h v_{F} / \tilde{g} \mu_{B} B_{x}$. When $\tilde{g} \mu_{B} B_{x} W / \hbar v_{F}=\pi / 2$ the first node of the order parameter occurs a distance $W$ from the superconductor. (b) As the magnitude of magnetic field increases, the wavelength of order parameter oscillations decreases. When $\tilde{g} \mu_{B} B_{x} W / \hbar v_{F}=3 \pi / 2$, the second order parameter node lies a distance $W$ from the superconductor. (c) If instead BIA dominates, the order parameter oscillations occur in the $x$ direction. As the magnetic field increases, the frequency of oscillations increases. In the inset, a linecut of the order parameter a distance $W$ from the superconductor demonstrates that oscillations are an end effect, with amplitudes which decay into the bulk of the 2DEG. (d) With weak spin-orbit coupling, the parallel field $B_{x}$ introduces order parameter oscillations in both directions. 

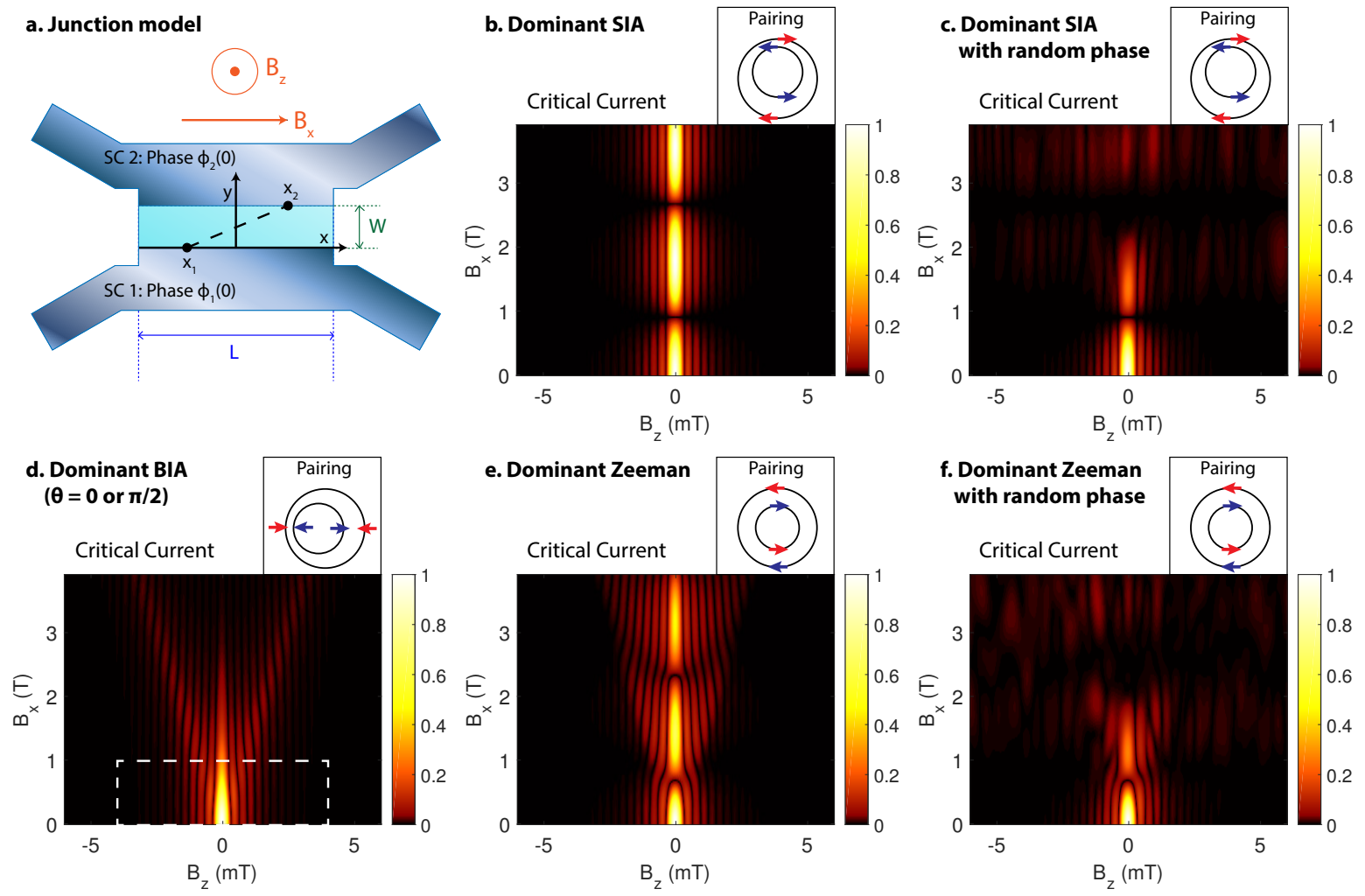

Figure 3: Modeling Josephson interference between two superconducting leads. (a) With two leads, paired electrons may traverse the junction beginning at a point $x_{1}$ in the lower superconducting lead (SC 1). The pairing amplitude at the point $x_{2}$ in the upper lead (SC 2), takes account of the phase accumulated due to finite pairing momentum within the HgTe quantum well. (b) With SIA dominant, the external magnetic field $B_{x}$ increases the pairing wavevector $\Delta k$ only in the $y$ direction. At certain values $\Delta k=(2 n+1) \pi /(2 W), n$ integer, the superconducting interference disappears. A diagram schematically depicts the expected Fermi surfaces and Cooper pairing, where arrows denote spin direction and pairs are each colored blue or red. Similar diagrams throughout this figure indicate the expectation for pairing and Fermi surfaces as the model parameters change. (c) Randomness at the interface between the quantum well and superconductors may arise due to structural imperfections. The random phase causes superconductivity to weaken and increases the width of nodes as the parallel field increases. (d) For junctions aligned to a principal crystal axis, dominant BIA leads to a pairing wavevector $\Delta k$ that grows in the $x$ direction as $B_{x}$ increases. The critical current maxima then occur at increasingly large values of $\left|B_{z}\right|$ as $B_{x}$ grows. Fabricating devices at varying angles with respect to the crystal is expected to modify the interference when BIA dominates. The region outlined in dashed white corresponds to the measured region in Figure 1e. (e) With dominant Zeeman coupling, the pairing magnitude is isotropic in-plane and the interference grows as a hybrid of the SIA and BIA cases. Characteristically, interference fringes repeatedly combine to form the central fringe at each successive node in the parallel field. Additionally, with zero perpendicular field, superconductivity disappears at values of parallel field that are smaller than the nodal magnetic field in the case with dominant SIA. (f) Including randomness leads to a similar picture to (c), while retaining the combining of fringes characteristic of dominant Zeeman coupling. 


\section{a. Niobium Leads}

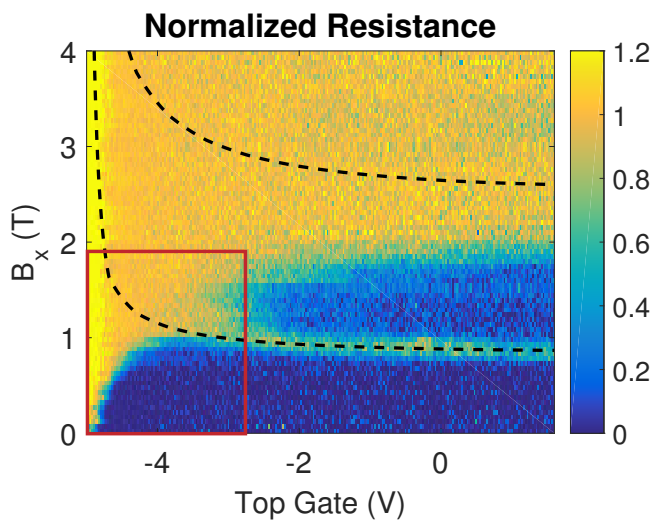

c. g-factor

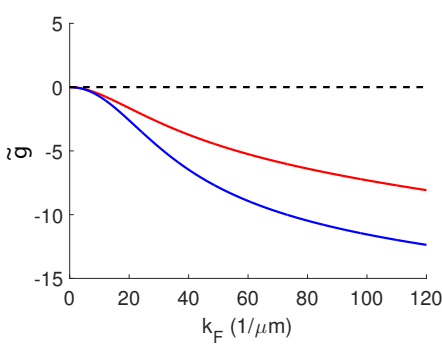

b. Aluminum Leads

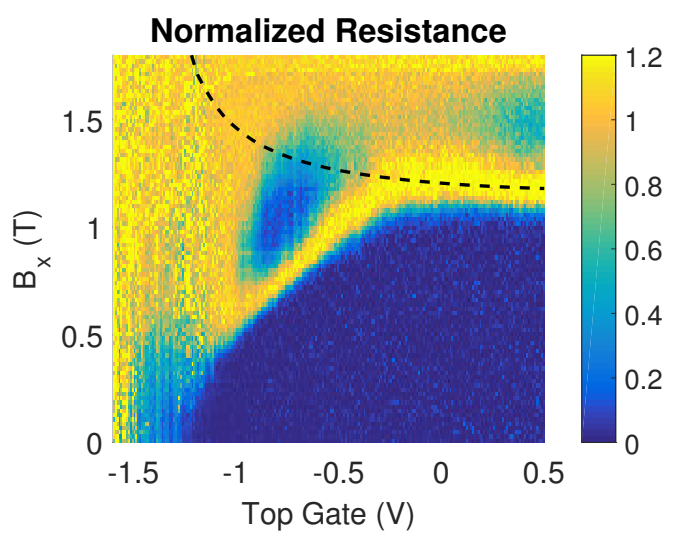

e. Pair momentum shift

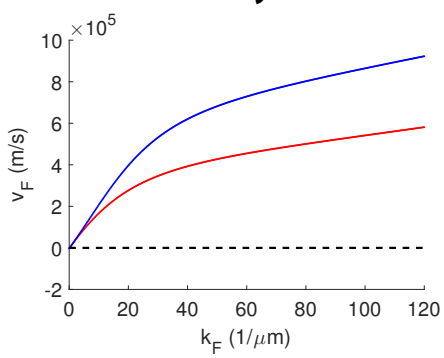

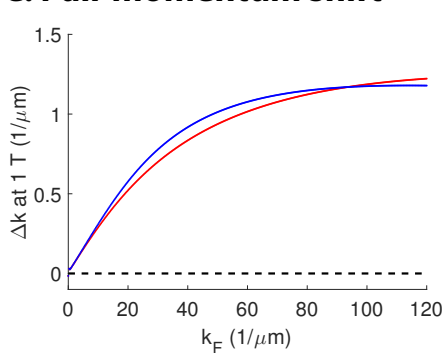

Figure 4: The evolution of minimum differential resistance as density and parallel magnetic field $B_{x}$ vary. Differential resistance measurements are normalized at each point by the normal junction resistance. (a) At the highest gate voltage in the niobium junction, increasing the magnetic field leads to periodic high-resistance nodes separating regions of decreased resistance. As the gate voltage is decreased, the magnetic field at the first node rises to larger values of $B_{x}$. (b) The aluminum junction behaves similarly to the niobium junction, although the measurement is limited to a smaller region outlined in red in (a). At low density, the magnitude of the nodal magnetic field begins to decrease as the density is lowered, a feature which remains to be understood. (c) The values of $B_{x}$ at which we expect nodes to appear are sensitive to the density dependence of both the in-plane g-factors and the Fermi velocities, calculated here assuming that SIA is due to an electric field of $10 \mathrm{mV} / \mathrm{nm}$. Blue and red curves correspond to the inner and outer Fermi surfaces, respectively. As the magnitude of the Fermi wavevector $k_{F}$ decreases, the in-plane g-factors shift from -20.5 toward zero. (d) Meanwhile the magnitudes of the Fermi velocities decrease to zero. (e) The pairing momenta induced at $1 \mathrm{~T}$ consequently decrease to zero from approximately $1.2 / \mu \mathrm{m}$. Since there can be no induced momentum at zero density, the nodal magnetic field diverges as the density is lowered. The nodal magnetic field, averaged over the two Fermi surfaces, is calculated using BHZ theory and plotted as dashed black lines in (a) and (b). 


\title{
Supplementary Information for Controlled Finite Momentum Pairing and Spatially Varying Order Parameter in Proximitized HgTe Quantum Wells
}

\author{
Sean Hart ${ }^{\dagger 1}$, Hechen Ren ${ }^{\dagger 1}$, Michael Kosowsky ${ }^{1}$, \\ Gilad Ben-Shach ${ }^{1}$, Philipp Leubner ${ }^{2}$, Christoph Brüne ${ }^{2}$, Hartmut Buhmann², $^{2}$ \\ Laurens W. Molenkamp ${ }^{2}$, Bertrand I. Halperin ${ }^{1}$, Amir Yacoby ${ }^{1}$ \\ ${ }^{1}$ Department of Physics, Harvard University, Cambridge, MA, USA \\ ${ }^{2}$ Physikalisches Institut (EP3), Universität Würzburg, 97074 Würzburg, Germany \\ †These authors contributed equally to this work
}

\section{Wafer characteristics and General Measurements}

Junctions were fabricated using $\mathrm{HgTe} / \mathrm{HgCdTe}$ heterostructures grown in the [001] crystal direction (the $z$ direction), composed as shown in Supplementary Figure 1. Wafer I contained an $8 \mathrm{~nm}$ quantum well with an electron density of $13.5 \times 10^{11} / \mathrm{cm}^{2}$ and a mobility of $390,000 \mathrm{~cm}^{2} /$ Vs. Wafer II contained a $7.8 \mathrm{~nm}$ quantum well with an electron density of $2.9 \times 10^{11} / \mathrm{cm}^{2}$ and a mobility of $790,000 \mathrm{~cm}^{2} / \mathrm{Vs}$. Josephson junctions fabricated on these wafers were aligned at varying angles with respect to the [110] and [110] cleavage edges of the crystal, but we do not know which is which in our samples. Therefore, we can only specify that the angular alignment corresponds to a rotation angle $\theta$ with respect to the [100] crystal axis, modulo $\pi / 2$. Although we do not know which principal axis $\theta$ is referenced to experimentally, our model predicts the same results when $\theta$ is referenced to either. The $x$ and $y$ axes always lie respectively perpendicular and parallel to the direction of current flow in devices (see Figure 1 of main text).

Throughout this supplement, devices are referred to in the following manner. Device A was fabricated by depositing aluminum leads onto a mesa etched into Wafer I, and was oriented at an angle $\theta=\pi / 4$. Devices B, C, and D were concurrently fabricated by depositing aluminum leads on Wafer II, and were respectively oriented at different angles $\theta=0, \pi / 2$, and $\pi / 4$. Device E was fabricated using wafer I, contained niobium leads, and was oriented at $\theta=\pi / 4$. Device $\mathrm{F}$ was fabricated using Wafer I, contained aluminum leads, and was oriented at $\theta=\pi / 4$. Note that data from devices $\mathrm{C}, \mathrm{D}$ and $\mathrm{E}$ were reported in the main text. 
Devices were processed as follows. To define the ends of the junctions, mesas $100 \mathrm{~nm}$ in height were etched using an argon ion source. To fabricate superconducting contacts, the contact area was etched enough to expose the quantum well using argon milling. Without breaking vacuum, the contact material was then deposited. For devices A-D and F, $5 \mathrm{~nm}$ of titanium was deposited by thermal evaporation, followed by thermal evaporation of $15 \mathrm{~nm}$ of aluminum. For device E, $10 \mathrm{~nm}$ of titanium was deposited by e-beam evaporation, followed by $130 \mathrm{~nm}$ of niobium deposited by DC magnetron sputtering. Next, a $50 \mathrm{~nm}$ layer of aluminum oxide was grown using atomic layer deposition, to isolate the final topgate layer (10 nm of titanium and $250 \mathrm{~nm}$ of gold) from the underlying junctions.

Measurements were performed primarily in a dilution refrigerator outfitted with a 6-1-1 vector magnet capable of applying up to $6 \mathrm{~T}$ in one direction, and $1 \mathrm{~T}$ in the two remaining directions. Experiments were carried out at the mixing chamber base temperature of $50 \mathrm{mK}$. Unless otherwise mentioned, all measurements occurred in this system. One measurement was performed in a separate dilution refrigerator with a base temperature of $10 \mathrm{mK}$.

As either the external magnetic field or the topgate voltage was varied, the differential four-terminal resistance of junctions was monitored using standard lock-in techniques. To determine critical currents or to provide bias sufficient to measure the normal resistance of the junctions, sometimes a DC current bias was applied to the junctions. Otherwise all measurements occurred with no DC current bias.

\section{Characterization of Thin Aluminum Leads}

The resistance of thin aluminum leads was characterized as a function of magnetic field in both the $x$ direction and the $y$ direction (Supplementary Figure 2). In each plot, red and blue color coding corresponds to the two leads of a single junction. Different junctions were used in the two plots, demonstrating the consistency of the fabrication process. The critical parallel field of the $15 \mathrm{~nm}$ aluminum films was consistently above $1.5 \mathrm{~T}$, in agreement with a previous study of thin aluminum [19]. The data presented in Supplementary Figure $2 \mathrm{~b}$ was collected at a temperature of $10 \mathrm{mK}$ in the system discussed previously.

\section{Critical Current of a Josephson Junction Under External Par- allel Magnetic Field}

Here we present measurements of the critical current in device D, for different values of the external magnetic field. When compared to corresponding measurements of differential resistance without any 
applied DC current (Supplementary Figure 4e and Supplementary Figure 5e), it is apparent that both measurement modes reveal the same basic behaviors.

In the measurements of critical current presented here, increasing the external field in either the $x$ or $y$ direction results in a decrease of the maximum critical current (Supplementary Figure 3a, b). This decrease occurs more rapidly for $B_{y}$, where the critical current becomes too small to reliably measure when $B_{y}$ exceeds 0.44 T. $B_{x}$, however, must exceed $1.1 \mathrm{~T}$ before critical currents become immeasurably small.

Upon normalization of the interference pattern at each separate value of parallel field, the asymmetry between the two directions becomes more pronounced (Supplementary Figure 3c, d). At all values of $B_{x}$, the shape of the Josephson interference remains essentially unaffected. By contrast, as $B_{y}$ increases the critical current splits into two separate maxima which occur at larger values of $\left|B_{z}\right|$. In Supplement XI we model the effect of the in-plane field $B_{y}$, which we expect to induce a finite $x$ component of the pairing wavevector that grows linearly with $B_{y}$. In junctions with finite length and with $B_{z}=0$, this pairing momentum in the $x$ direction leads to oscillations in the order parameter which are most pronounced near the ends of the junction. As a consequence, we expect that as $B_{y}$ increases, the maximum critical currents in our junctions will occur at values of $\left|B_{z}\right|$ that grow linearly with $B_{y}$. With only the parallel field $B_{x}$ present, however, the induced pair momentum is expected to lie along the $y$ direction. In this case no such wavevector in the $x$ direction is observed.

\section{Josephson Junctions Rotated with Respect to the Crystal}

Measurements of the differential resistance were performed on junctions oriented at different angles with respect to the crystal lattice, in order to determine whether bulk inversion asymmetry (BIA) plays a significant role in the momentum acquired by Cooper pairs. As previously mentioned, these devices A-E have orientation $\theta=\pi / 4,0, \pi / 2, \pi / 4$, and $\pi / 4$ respectively. Devices A-D use aluminum leads, while device $\mathrm{E}$ uses niobium leads. For each set of the devices, we explored behaviors resulting from a parallel magnetic field applied in the $x$ direction or in the $y$ direction, which in the previous section were shown for device $\mathrm{D}$ to differ.

Even as the angle $\theta$ varies among devices, the manner in which superconductivity evolves due to the applied field $B_{x}$ remains qualitatively unchanged at high density (Supplementary Figure 4). Devices $\mathrm{B}-\mathrm{D}$, fabricated on a single piece of wafer, show quantitative agreement in the value of $B_{x}$ at which a superconducting node appears. Devices A and E were separately fabricated on Wafer I, and show slight quantitative differences but nevertheless the same shape. The appearance of these nodes 
in the interference evolution, with no dependence on the crystal orientation, signals that structural inversion asymmetry (SIA) dominates the behavior of our quantum wells in the electron-doped regime (Supplement VI-IX).

With the parallel field applied in the $y$ direction and at high density, the interference pattern splits, forming a ' $\mathrm{V}$ ' shape as $B_{y}$ increases that is qualitatively identical for all values of $\theta$ (Supplementary Figure 5). The slope of the two arms of the ' $\mathrm{V}$ ' varies among devices, but is similar for devices B-D which were fabricated concurrently. The most dramatic effect is seen for device E with $130 \mathrm{~nm}$ thick niobium leads, in which the slope is approximately 7 times smaller than the other devices.

From the above measurements one can conclude that the basic differences in interference as $B_{x}$ or $B_{y}$ is increased have little to do with the orientation of the crystal lattice. The most striking difference is found among the data with the magnetic field oriented along the $y$ direction, in which the thickness of the leads correlates to the slope of the interference splitting. This behavior, which results from magnetic flux penetrating the area $d L$ formed by the length $L$ of the junction and the height difference $d$ between the center of the quantum well and the center of the leads, is modeled in Supplement XI.

\section{Evolution of Interference Lobes as the Parallel Magnetic Field $B_{x}$ Increases, in a Device with $\theta=\pi / 2$}

In the previous section, it was observed that at particular values of the parallel magnetic field $B_{x}$, nodes of suppressed superconductivity occur in our Josephson junctions. Additionally, this evolution of the Josephson interference with the parallel field $B_{x}$ does not depend on the device orientation with respect to the crystal. The appearance of nodes with lack of $\theta$ dependence already suggests that the effect of bulk inversion asymmetry (BIA) in our devices is small, and that structural inversion asymmetry (SIA) dominates (Supplement VI-IX). Still, the evolution of critical currents, in particular the maximum critical current of interference lobes occurring at nonzero perpendicular field, can provide further evidence that BIA is weak. When $\theta=\pi / 2$, in the limit of strong BIA these side lobe maximum critical currents are expected to grow as the parallel field $B_{x}$ is increased from zero (Figure 3d of main text). However, if SIA is strong, these critical currents are expected to monotonously decay as the parallel field increases up to the first node.

In Device $\mathrm{C}$, oriented at $\theta=\pi / 2$, we study the evolution of the first three side lobes adjacent to the central lobe (Supplementary Figure 6a). A measurement of the critical currents of these lobes indicates that all lobes are largest when $B_{x}=0 \mathrm{~T}$ (Supplementary Figure 6b). We extract the maximum critical 
current for each side lobe, plotted in Supplementary Figure 6c for each lobe. All critical currents are largest when $B_{x}=0 \mathrm{~T}$, and all decay monotonously until becoming indistinguishable from zero. This evolution of the side lobe critical currents provides additional evidence consistent with weak BIA in our devices.

\section{Four-Band Model and Spin-Orbit Effects in the Quantum Well}

A four-band model has been developed starting from $k \cdot p$ theory, and subsequently used to describe the topology of HgTe quantum wells [4. Here we adopt an elaboration on this model, where bulk inversion asymmetry (BIA), structural inversion asymmetry (SIA), and coupling to an external magnetic field are included. The four bands originate in the $s$ - and $p$-like bands of the underlying crystals, so that the basis states are written as $\left|E 1, m_{J}=+1 / 2\right\rangle,\left|H 1, m_{J}=+3 / 2\right\rangle,\left|E 1, m_{J}=-1 / 2\right\rangle$, and $\left|H 1, m_{J}=-3 / 2\right\rangle$. In this notation, $E 1$ refers to electron-like states with angular momentum $1 / 2$, while $H 1$ refers to holelike states with angular momentum 3/2. The Hamiltonian describing the system is then [23, 24, 25]:

$$
\begin{gathered}
H_{1}=\epsilon\left(\hat{k}^{2}\right)+M\left(\hat{k}^{2}\right) s_{z}+A \hat{k}_{x} s_{x} \sigma_{z}-A \hat{k}_{y} s_{y}+h\left(\cos \left(2 \theta^{t h}\right) \sigma_{y}+\sin \left(2 \theta^{t h}\right) \sigma_{x}\right) s_{y} \\
+R_{0} \frac{s_{z}+1}{2}\left(\hat{k}_{x} \sigma_{y}-\hat{k}_{y} \sigma_{x}\right)-\mu_{B} \cdot \vec{B} \cdot \vec{M}
\end{gathered}
$$

where

$$
\epsilon\left(\hat{k}^{2}\right)=C-D\left(\hat{k}_{x}^{2}+\hat{k}_{y}^{2}\right), M\left(\hat{k}^{2}\right)=M-B\left(\hat{k}_{x}^{2}+\hat{k}_{y}^{2}\right)
$$

and

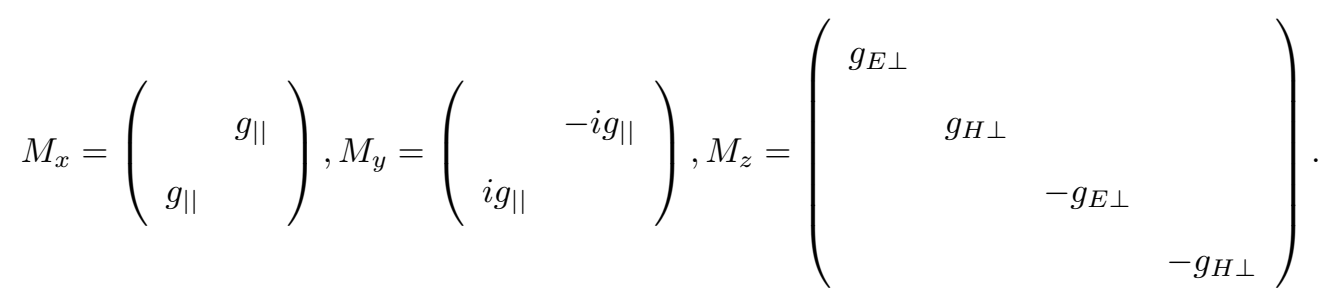

The first four terms are those present in the original theory of Bernevig, Hughes, and Zhang. The fifth term describes the magnitude $h$ of the BIA and the angle $\theta^{\text {th }}$ between the $x$ axis and the [100] crystal direction. The sixth term includes SIA with strength $R_{0} /(e \mathcal{E})$, where $\mathcal{E}$ is the magnitude of an electric field oriented along the $z$ axis. Coupling to the external magnetic field occurs anisotropically and is different for $E 1$ and $H 1$ states due to the inability of magnetic field to couple $m_{J}= \pm 3 / 2$ to first order. The value of the parallel g-factor $g_{\|}$and perpendicular g-factors $g_{E \perp}$ and $g_{H \perp}$, along with all 
other parameter values, are listed in Table 1 . The in-plane wavevectors are $\hat{k}_{x}=i \partial_{x}$ and $\hat{k}_{y}=i \partial_{y}$.

The bare effect of a parallel magnetic field is visualized by setting $h=0$ and $R_{0}=0$ and calculating the conduction band spectrum, under various values of $\vec{B}$ (Supplementary Figure 7a-c). In each plot, the bands are plotted up to the Fermi energy, which varies in order to demonstrate various limiting cases of weak or strong spin-orbit coupling. The Fermi surfaces are projected onto a plane below the bands, with the spin texture plotted at various points on the Fermi surfaces. The $x$ and $y$ components of the spin vectors are equal respectively to $\left\langle\sigma_{x}\left(1+s_{z}\right) / 2\right\rangle$ and $\left\langle\sigma_{y}\left(1+s_{z}\right) / 2\right\rangle$, the spin expectation values projected onto the $E 1$ bands.

With no external magnetic field, the conduction band is doubly degenerate due to the presence of the spin degree of freedom, modeled here up to a Fermi energy equal to $20 \mathrm{meV}$. Changing the magnetic field to $3 \mathrm{~T}$ in the $x$ direction lifts this degeneracy due to the Zeeman effect, resulting in two concentric Fermi surfaces centered at $\vec{k}=0$. Within the outer Fermi surface, spins point toward $-x$, due to the negative value of $g_{\|}$. Within the inner Fermi surface, spins are oppositely oriented. Application of a $3 \mathrm{~T}$ magnetic field in the $y$ direction identically splits the bands, although the spins in this case orient along $\pm y$. It can be seen that with no spin-orbit coupling and an applied in-plane magnetic field, pairs may form with nonzero wavevector in any in-plane direction.

Including dominant SIA (equivalent to a perpendicular electric field of $10 \mathrm{mV} / \mathrm{nm}$ ) with no BIA modifies the expectation for the band structure (Supplementary Figure 7d-f). Even without any external magnetic field, in this case the bands are spin-split for all nonzero wavevectors. The axial symmetry of the spin-orbit coupling results in the spin texture shown in Supplementary Figure 7e, modeled up to a higher Fermi energy of $50 \mathrm{meV}$ to highlight the limit of strong SIA. Now coupled with an external magnetic field, these factors result in a behavior that is different from the case with no spin-orbit coupling. When magnetic field is applied in the $x(y)$ direction, the two Fermi surfaces shift oppositely in the $y(x)$ direction. Consistent with our observations on aluminum-basd junctions in the electrondoped regime, this shift implies that a magnetic field in the $x$ direction introduces a pairing wavevector in the $y$ direction. Also superficially consistent with our data, a magnetic field in the $y$ direction leads to a pairing wavevector in the $x$ direction. However, the magnitude of this wavevector alone is too small to explain the experimental evolution of interference as $B_{y}$ is increased. A full explanation of this effect is presented in Supplementary Information XI. Finally, the lack of dependence of SIA on the angle $\theta^{t h}$ is consistent with our measurements (Supplementary Figures 4 and 5).

In the remaining case, the effect of dominant BIA with no SIA is investigated in Supplementary Figure $7 \mathrm{~g}-\mathrm{i}$, for an angle $\theta^{\text {th }}=0$ and up to a Fermi energy of $20 \mathrm{meV}$. Similarly to the case of strong SIA, with no external magnetic field present the bands are spin-split at nonzero wavevectors. However, 
the texture of spins at the Fermi surfaces displays tetrahedral symmetry in this case. As a result, when parallel magnetic field is present in the $x(y)$ direction, the two Fermi surfaces shift oppositely in the $x$ (y) direction. This shifting is orthogonal to the shifts present with strong SIA, and does not agree with our interference measurements on devices aligned with $\theta=0$ or $\pi / 2$. Furthermore, the shift direction rotates by $\pi / 2$ as the angle $\theta^{\text {th }}$ becomes $\pi / 4$. This prediction that the direction of induced Cooper pair momentum should depend on $\theta^{\text {th }}$ is also inconsistent with our results.

Finally, in the main text, we model the evolution with density of the in-plane g-factor $\tilde{g}$, the Fermi velocity $v_{F}$, and the pair momentum shift $\Delta k \approx \tilde{g} \mu_{B} B_{x} / \hbar v_{F}$, under the assumption that BIA is absent and SIA is dominant (equivalent to a perpendicular electric field of $10 \mathrm{mV} / \mathrm{nm}$ ). At each density, a particular value of the magnetic field satisfies the condition $\Delta k W=\pi / 2$, leading to a node in the induced superconductivity in the junction. We find that the evolution with density of this nodal magnetic field value agrees with our model at high densities. In niobium and aluminum devices respectively, the value of the nodal magnetic field is consistent with values of of $\tilde{g} / v_{F}$ that are approximately 1.9 and 1.4 times greater than those expected theoretically. Using different values for the electric field only weakly modifies this conclusion. For example, if the SIA was instead equivalent to a perpendicular electric field of $40 \mathrm{mV} / \mathrm{nm}$, we would find values of of $\tilde{g} / v_{F}$ approximately 2.1 and 1.5 times greater than the theoretical expectation, only a $\sim 10 \%$ difference.

\section{Model of a Two-Dimensional Electron Gas Contacted by Su- perconducting Leads}

In the following sections, we model the coupling of superconducting leads to our quantum well. We consider a geometry in which a two-dimensional electron gas (2DEG) is contacted by a pair of superconducting leads with a controlled phase difference between them, and we seek to calculate the maximum supercurrent that can be carried between the strips. The following is a more complete derivation of the pair propagator $F$ used in the main text to carry out this goal. We assume a Hamiltonian $H=H_{0}+H_{2}$, where $H_{0}$ is the Hamiltonian for the 2DEG in the absence of the superconductor, and $H_{2}$ is the coupling between the superconductors and the 2DEG, described by a pairing Hamiltonian of the form

$$
H_{2}=-\int d x d y\left[\Delta(x, y) \Psi^{\dagger}(x, y)+\Delta^{*}(x, y) \Psi(x, y)\right]
$$

Here $\Psi(x, y) \equiv \psi_{\uparrow}(x, y) \psi_{\downarrow}(x, y)$ is an operator which annihilates a singlet pair of electrons in the 2DEG at the point $(x, y)$, while the pair potential $\Delta(x, y)$ is a complex number that depends on the phase of 
the superconductor and the tunneling amplitude at that point.

We assume that the contacts between the 2DEG and the superconductors occur at the edges of the superconductors, located at $y=0$ and $y=W$, so that we may write

$$
\Delta(x, y)=\lambda_{1}(x) \delta(y)+\lambda_{2}(x) \delta(y-W) \equiv \Delta_{1}(x, y)+\Delta_{2}(x, y)
$$

with $-L / 2<x<L / 2$. We assume that the magnitude of the coupling is constant along each lead, but the phase will vary if there is a perpendicular magnetic field $B_{z} \neq 0$. We choose a gauge where the vector potential points in the $x$ direction, with $A_{x}=-B_{z}(y-W / 2)$, so that the vector potential vanishes along the midline of the $2 \mathrm{DEG}$. If the superconducting strips have identical widths $W_{S C}$, then the couplings $\lambda_{j}$ will have the form

$$
\begin{gathered}
\lambda_{j}(x)=\left|\lambda_{j}\right| e^{2 \pi i \phi_{j}(x)}, \\
\phi_{j}(x)=\phi_{j}(0)+\frac{(-1)^{j-1} x B_{z}\left(W+W_{s c}\right)}{2 \Phi_{0}},
\end{gathered}
$$

with $j=1,2$. The phases in equation (15) are determined by the condition that there should be no net current flow along the length of the superconducting strips, so the phase gradient in each superconductor should be canceled by the vector potential along the center line of the superconductor.

We assume that $B_{z}$ is sufficiently weak that the cyclotron radius $R_{c}=\hbar k_{F} / e B_{z}$ is large compared to $W$ (typically in our devices $R_{c} \approx 10 \mu \mathrm{m}$, which is large compared to $W=800 \mathrm{~nm}$ ). In this case, we may ignore the effect of $B_{z}$ on the trajectories of electrons in the 2DEG. Moreover, since we have chosen the vector potential to vanish along the midline of the $2 \mathrm{DEG}$, an electron crossing from $y=0$ to $y=W$ will acquire no net phase due to the vector potential. We also ignore, for the moment, any orbital effects of the parallel field $B_{\|}$. Thus the 2DEG Hamiltonian $H_{0}$ will include the Zeeman coupling to $B_{\|}$, as well as the spin-orbit coupling, but will not include terms due to the magnetic field in the kinetic energy.

To lowest order in the pairing Hamiltonian $\mathrm{H}_{2}$, the portion of the total energy that depends on the phase difference between the two superconducting leads can be written in the form:

$$
E=-\int d x_{2}\left[\lambda_{2}^{*}\left(x_{2}\right)\left\langle\Psi\left(x_{2}, W\right)\right\rangle_{1}+\text { c.c. }\right]
$$

where $\langle\Psi(x, y)\rangle_{1}$ is the order parameter at point $(x, y)$ induced by the superconductor $j=1$. In turn, this may be written in the form 


$$
\langle\Psi(x, y)\rangle_{1}=\int d x_{1} \lambda_{1}\left(x_{1}\right) F\left(x, x_{1}, y\right)
$$

where $F$ is the propagator from point $\left(x_{1}, 0\right)$ to point $(x, y)$ for an induced Cooper pair. We will determine the form of $F$ in the following section.

As a first approximation, we may ignore the fact that there are boundaries of the 2DEG at $x= \pm L / 2$ and that electrons will be reflected at these boundaries (either specularly or diffusely, and possibly with a spin flip). Similarly, we ignore the possibility of single-particle reflection at $y=0$ or $y=W$, where the superconducting leads touch the 2DEG. Furthermore, we assume that the electron density is constant in the 2DEG, and we ignore any interactions between electrons in the 2DEG. We also ignore scattering by impurities inside the 2DEG. Then the propagator $F$ may be calculated for an infinite, translationally invariant $2 \mathrm{DEG}$, where the momentum of each electron is a good quantum number. We believe that corrections due to reflections at the boundaries will have quantitative effects but will not affect qualitatively the form of our results. Modeling of critical current including specular reflections at mesa edges will be discussed in Supplement X and XI.

\section{Derivation of a General Formula for the Pair Propagator $F$}

The four-band model Hamiltonian $H_{1}$ for our quantum wells was previously discussed. Since we presently are interested in behavior near the Fermi energy $E_{F}$, and within the conduction bands only, in the following we adopt a simplified two-band model Hamiltonian. This Hamiltonian for an electron in the 2DEG with momentum $\vec{k}$ is given by the $2 \times 2$ matrix

$$
\begin{gathered}
H_{\vec{k}}^{0}=v_{F}\left(k-k_{F}\right)+\vec{\beta}(\hat{k}) \cdot \vec{\sigma}, \\
\beta_{j}=\tilde{g} \mu_{B} B_{j} / 2+\hat{k}_{i} S_{i j},
\end{gathered}
$$

where $B_{j}$ are the $x$ and $y$ components of the in-plane magnetic field, $\tilde{g}$ is an effective g-factor, $\hat{k} \equiv \vec{k} / k$, and $\hat{k}_{i} S_{i j} \sigma_{j}$ is the spin-orbit coupling term, which we assume to be small compared to the Fermi energy. We have here assumed a single electron band, and assumed that band structure is isotropic in the absence of spin-orbit coupling. We can then write

$$
H_{\vec{k}}^{0}=\sum_{\eta} \epsilon_{\vec{k} \eta} P^{\hat{k} \eta}
$$


with $\eta= \pm 1$. Here $\epsilon_{\vec{k} \eta}$ are the two eigenvalues, and $P^{\hat{k} \eta}$ are projection matrices given by

$$
\epsilon_{\vec{k} \eta}=v_{F}\left(k-k_{F}\right)+\eta|\vec{\beta}|, P^{\hat{k} \eta}=(1+\eta \hat{\beta} \cdot \vec{\sigma}) / 2,
$$

with $\hat{\beta} \equiv \vec{\beta} /|\vec{\beta}|$.

We next define a $2 \times 2$ matrix function

$$
g(\vec{r}, \epsilon) \equiv \frac{1}{(2 \pi)^{2}} \sum_{\eta} \int d^{2} k e^{i \vec{k} \cdot \vec{r}} \delta\left(\epsilon-\epsilon_{\vec{k} \eta}\right) P^{\hat{k} \eta}
$$

Then, letting $\vec{r}=\left(x-x_{1}, y\right)$, the pair propagator $F\left(x, x_{1}, y\right)$ may be expressed as

$$
F(\vec{r})=\int_{0}^{\infty} d \epsilon \int_{0}^{\infty} d \epsilon^{\prime} \frac{\operatorname{tr}\left[g(\vec{r}, \epsilon) \sigma^{y} g^{T}\left(\vec{r}, \epsilon^{\prime}\right) \sigma^{y}+g(\vec{r},-\epsilon) \sigma^{y} g^{T}\left(\vec{r},-\epsilon^{\prime}\right) \sigma^{y}\right]}{2\left(\epsilon+\epsilon^{\prime}\right)}
$$

where $T$ indicates the matrix transpose.

We are interested in the situation where $k_{F} r \gg 1$, and $|\epsilon| \ll E_{F}$. Then the integration over the direction of $\vec{k}$ is dominated by regions close to the end points where $\vec{k}$ is either parallel or antiparallel to $\vec{r}$, and the expression for $g(\vec{r}, \epsilon)$ may be approximated by

$$
g(\vec{r}, \epsilon) \approx \frac{k_{F}^{1 / 2}}{(2 \pi)^{3 / 2} v_{F} r^{1 / 2}} \sum_{\eta}\left[e^{i k_{F} r} e^{i \epsilon r / v_{F}} e^{-i \pi / 4} e^{-i \delta k_{\eta}^{+} r} P^{\eta+}+e^{-i k_{F} r} e^{-i \epsilon r / v_{F}} e^{i \pi / 4} e^{i \delta k_{\eta}^{-} r} P^{\eta-}\right]
$$

where $P^{\eta \pm}$ is equal to $P^{\hat{k} \eta}$, with $\hat{k}= \pm \hat{r} \equiv \pm \vec{r} / r$ and

$$
\delta k_{\eta}^{ \pm}=\frac{\eta|\vec{\beta}( \pm \hat{r})|}{v_{F}} .
$$

When we substitute the expression for $g$ in formula $(23)$ for $F(\vec{r})$, we may ignore the terms proportional to $e^{ \pm 2 i k_{F} r}$, as these rapidly oscillating terms will give vanishing contribution to the energy if the width of the contacts between the 2DEG and the superconducting strips are large compared to $1 / k_{F}$. Performing the integrals over $\epsilon$ and $\epsilon^{\prime}$ in the remaining terms, one obtains the result

$$
F(\vec{r}) \approx \frac{C}{r^{2}} \sum_{\eta \eta^{\prime}} N_{\eta \eta^{\prime}}(\hat{r}) e^{-i\left(\delta k_{\eta}^{+}-\delta k_{\eta^{\prime}}^{-}\right) r}
$$

with $C=k_{F} /\left(8 \pi^{2} v_{F}\right)$, and

$$
N_{\eta \eta^{\prime}}(\hat{r})=N_{\eta \eta^{\prime}}(-\hat{r})=\frac{1-\eta \eta^{\prime} \hat{\beta}(\hat{r}) \cdot \hat{\beta}(-\hat{r})}{2} .
$$




\section{Special Cases and Limiting Forms of the Pair Propagator}

Here we discuss several special cases which lead to limiting forms for the pair propagator $F(\vec{r})$. The above expressions (equations (26) and (27)) may be simplified in the limit where the Zeeman energy is small compared to the spin-orbit energy splitting. When $B_{\|}=0$, we find that $\hat{\beta}(\hat{r})=-\hat{\beta}(-\hat{r})$, so that $N_{\eta \eta^{\prime}}=\delta_{\eta \eta^{\prime}}$. Furthermore, when $\eta=\eta^{\prime}$, we see that the exponent in equation (26) is equal to zero, so $F$ will have no oscillations as a function of $r$. If $B_{\|}$is nonzero but still small compared to the spin-orbit splitting, it remains a good approximation to set $N_{\eta \eta^{\prime}}=\delta_{\eta \eta^{\prime}}$. In the exponent, however, we have

$$
\left(\delta k_{\eta}^{+}-\delta k_{\eta}^{-}\right)=\eta \tilde{g} \mu_{B} \vec{B}_{\|} \cdot \vec{\beta}(\vec{r}) .
$$

An important example is the case of pure SIA spin-orbit coupling, where the matrix $S$ has the Rashba form, $S \propto i \tau^{y}$, where $\tau^{y}$ is a Pauli matrix. In this case we may write

$$
\left(\delta k_{\eta}^{+}-\delta k_{\eta}^{-}\right) r=\eta \Delta \vec{k} \cdot \vec{r}
$$

with

$$
\Delta \vec{k}=\hat{z} \times \vec{B}_{\|} \tilde{g} \mu_{B} / v_{F}
$$

The oscillations in $F(\vec{r})$ have a simple interpretation in this case. When $B=0$, the Fermi surface consists of two circles centered about the origin, split by the spin-orbit coupling, with spin orientations shown in Figure 3b of the main text. Application of a weak in-plane magnetic field will shift the two Fermi circles in opposite directions, by amounts $\pm \Delta \vec{k} / 2$. The function $F(\vec{r})$ describes the propagator when a singlet pair of electrons is injected at one point and removed at a second point, separated by $\vec{r}$. For large separations, $F(\vec{r})$ is dominated by pairs of electrons that are close to the Fermi energy, with wave vectors opposite to each other and parallel or antiparallel to $\vec{r}$. Because the two electrons must have opposite spins, they must belong to the same branch of the Fermi surface. Thus, the induced pairs will have total momenta equal to $\pm \Delta \vec{k}$, depending on the branch $\eta$. The momentum shifts are manifest in the phase factors $e^{i \eta \Delta \vec{k} \cdot \vec{r}}$, which appear in $F(\vec{r})$ in this case.

In the case of pure BIA coupling, the matrix $S$ is $\propto \tau^{z}$, in our coordinate system. We may again write the phase accumulation in the form (29), but now the direction of $\Delta \vec{k}$ depends on the directions of $\vec{B}_{\|}$relative to the crystal axes.

The formula for $F(\vec{r})$ also becomes simple in the case where the Zeeman energy is large compared to the spin-orbit splitting. In this case, the Fermi surface consists of two concentric circles, with spin 
that are uniformly aligned on each circle, either parallel or antiparallel to $\vec{B}_{\|}$. In order to form a spin singlet, we must choose one electron from each Fermi circle. If we also require that the momenta be parallel or antiparallel to $\vec{r}$, we see that the induced electron pair will have a total momentum equal to $\pm \hat{r} \tilde{g} \mu_{B} B_{\|} / v_{F}$. Thus we should find that the phase shift is independent of the direction of $\vec{r}$.

These expectations may be confirmed using the formulas derived above. In the case where the Zeeman energy is large compared to the spin-orbit splitting, we find that $\vec{\beta}(\hat{k})$ is independent of $\hat{k}$, and thus $N_{\eta \eta^{\prime}}=\delta_{\eta,-\eta^{\prime}}$. Furthermore, $\delta k_{\eta}^{+}-\delta k_{-\eta}^{-}=\eta \tilde{g} \mu_{B} B_{\|} / v_{F}$, independent of $\vec{r}$.

\section{Reflections from the Sample Edges}

Taking into account the effects of electron reflections from the ends of the sample, at $x= \pm L / 2$, we should rewrite the propagator $F$ in a more general form as

$$
F\left(x_{2}, x_{1}, W\right)=F_{0}(\vec{r})+F_{1}\left[\left(x_{2}+\frac{L}{2}\right),\left(x_{1}+\frac{L}{2}\right), W\right]+F_{2}\left[\left(\frac{L}{2}-x_{2}\right),\left(\frac{L}{2}-x_{1}\right), W\right],
$$

where $F_{0}$ is the function given by equation (26) for the infinite system, $\vec{r}=\left(x_{2}-x_{1}, W\right)$, as before, and $F_{1}$ and $F_{2}$ describe the contributions of electrons reflected from the left boundary or right boundary respectively. We assume that the length $L$ is long enough that we can neglect the effects of electrons that scatter multiple times from opposite boundaries. Here we will assume that the boundaries at $x= \pm L / 2$ are represented by infinite potential barriers, which are perfectly smooth, so that electrons are specularly reflected with no change in spin. The symmetry of our problem will then be such that $F_{1}$ and $F_{2}$ have identical functional forms, so we need only find the form of $F_{1}$. For convenience, we move the left boundary to the line $x=0$, and we assume that the right boundary is located at $x=\infty$. Using similar reasoning to what we used in the translationally invariant case, we may write $F_{1}$ in the form

$$
F_{1}\left(x_{2}, x_{1}, W\right)=\int_{0}^{\infty} d \epsilon \int_{0}^{\infty} d \epsilon^{\prime} \frac{\operatorname{tr}\left[h\left(x_{2}, x_{1}, W, \epsilon\right) \sigma^{y} h^{T}\left(x_{2}, x_{1}, W, \epsilon^{\prime}\right) \sigma^{y}+c . c .\right]}{2\left(\epsilon+\epsilon^{\prime}\right)}
$$

where

$$
\begin{aligned}
h\left(x_{2}, x_{1}, W, \epsilon\right) & =-\frac{k_{F}^{1 / 2}}{(2 \pi)^{3 / 2} v_{F} s^{1 / 2}} \sum_{\eta_{1}, \eta_{2}}\left[e^{i k_{F} s} e^{i \epsilon s / v_{F}} e^{-i \pi / 4} e^{-i\left(\delta k_{1, \eta_{1}}^{+} s_{1}+\delta k_{2, \eta_{2}}^{+} s_{2}\right)} P_{1}^{\eta_{1}+} P_{2}^{\eta_{2}+}\right. \\
& \left.+e^{-i k_{F} s} e^{-i \epsilon s / v_{F}} e^{i \pi / 4} e^{i\left(\delta k_{1, \eta_{1}}^{-} s_{1}+\delta k_{2, \eta_{2}}^{-} s_{2}\right)} P_{1}^{\eta_{1}-} P_{2}^{\eta_{2}-}\right]
\end{aligned}
$$

where $s=\left[\left(x_{1}+x_{2}\right)^{2}+W^{2}\right]^{1 / 2}, s_{1}=s x_{1} /\left(x_{1}+x_{2}\right), s_{2}=s-s_{1}$, and 


$$
\delta k_{j, \eta}^{ \pm}=\frac{\eta\left|\vec{\beta}\left( \pm \hat{k}_{j}\right)\right|}{v_{F}},
$$

for $j=1,2$, with

$$
\hat{k}_{1}=-\frac{\left[\left(x_{1}+x_{2}\right),-W\right]}{s}, \hat{k}_{2}=\frac{\left[\left(x_{1}+x_{2}\right), W\right]}{s} .
$$

Furthermore, we have

$$
P_{j}^{\eta \pm}=\left(1+\eta \hat{\beta}\left( \pm \hat{k}_{j}\right) \cdot \vec{\sigma}\right) / 2 .
$$

We now turn to one particular example. In order to evaluate expression (32) for $F_{1}$, we must first evaluate the trace over a product of projection matrices and $\sigma^{y}$. In the case of strong SIA coupling and weak magnetic field, the trace simplifies, and we obtain the result

$$
\operatorname{tr}\left[P_{1}^{\eta_{1}+} P_{2}^{\eta_{2}+} \sigma^{y}\left(P_{1}^{\eta_{3}-} P_{2}^{\eta_{4}-}\right)^{T} \sigma^{y}\right]=\delta_{\eta_{1} \eta_{3}} \delta_{\eta_{2} \eta_{4}}\left[\sin ^{2} \theta \delta_{\eta_{1} \eta_{2}}+\cos ^{2} \theta \delta_{\eta_{1},-\eta_{2}}\right],
$$

where $\sin \theta=W / s$. Furthermore for $B_{\|}$in the $y$ direction, we find

$$
\left(\delta k_{j, \eta}^{+}-\delta k_{j, \eta}^{-}\right) s_{j}=(-1)^{j} \eta x_{j} \frac{\tilde{g} \mu_{B} B_{y}}{v_{F}} .
$$

Thus, in the case of strong SIA and $B_{\|}$in the $y$ direction, we find

$$
F_{1}\left(x_{2}, x_{1}, W\right)=\frac{2 C\left[\sin ^{2} \theta \cos \Delta k\left(x_{1}-x_{2}\right)+\cos ^{2} \theta \cos \Delta k\left(x_{1}+x_{2}\right)\right]}{\left(x_{1}+x_{2}\right)^{2}+W^{2}},
$$

where $\Delta k=\tilde{g} \mu_{B} B_{x} / v_{F}$, and the constant $C$ is the same as in equation (26).

\section{Modeling Josephson Interference}

Using the pair propagator $F(\vec{r})$ and equation (16), we can calculate the Josephson energy and critical current for our junctions. In the limit of either strong BIA or strong SIA, the Cooper pair momentum shift occurs at an angle $\alpha$ with respect to the $x$ axis and the pair propagator is

$$
F\left(x_{2}, x_{1}, W\right)=\frac{k_{F}}{8 \pi^{2} v_{F}} \cdot \frac{e^{i \gamma}+e^{-i \gamma}}{\left(x_{2}-x_{1}\right)^{2}+W^{2}}, \gamma=\Delta k\left(\sin (\alpha) W+\cos (\alpha)\left(x_{2}-x_{1}\right)\right) .
$$

As previously noted, in this case pairing occurs internally to each Fermi surface. In the limit of weak 
spin-orbit coupling, the pair propagator instead takes the form

$$
F\left(x_{2}, x_{1}, W\right)=\frac{k_{F}}{8 \pi^{2} v_{F}} \cdot \frac{e^{i \gamma}+e^{-i \gamma}}{\left(x_{2}-x_{1}\right)^{2}+W^{2}}, \gamma=\Delta k \sqrt{W^{2}+\left(x_{2}-x_{1}\right)^{2}} .
$$

Due to the opposite spin polarization of the two Fermi surfaces, pairing in this limit is expected to occur between Fermi surfaces, in contrast to the limit of large spin-orbit coupling.

The Josephson energy $E$ is obtained in each limit by evaluating equation (16). By differentiating the Josephson energy with respect to the phase difference $\phi_{1}(0)-\phi_{2}(0)$ we find the current-phase relation of the junction, which is then maximized with respect to the phase difference to obtain the critical current.

In the main text we consider only a parallel field along the $x$ direction, and we model the effect of structural imperfections in the interface between the superconducting leads and the quantum well. These would introduce random fluctuations in the direction of the in-plane magnetic field at each interface, leading to a random component of the pairing momentum in the $x$ direction that grows linearly with the in-plane field. Hence, we introduce a random phase $\chi \propto\left(R_{1}\left(x_{1}\right)-R_{2}\left(x_{2}\right)\right) B_{x}$, where the random variables $R_{1}\left(x_{1}\right)$ and $R_{2}\left(x_{2}\right)$ correspond to fluctuations in the direction of the parallel field at each interface. The modeled step size in $x$ is $40 \mathrm{~nm}$, with no correlations between adjacent positions. The random phase $\chi$ is uniformly distributed between zero and an upper bound whose absolute magnitude is equal to $15 \%$ of the maximum phase generated by the intrinsic momentum. With this randomness, the calculated critical currents diminish in magnitude as the in-plane field increases, and nodes occur over a larger range of in-plane field, in agreement with the experimental observation (shown in Figure $3 \mathrm{c}$ of the main text for the case of dominant SIA).

In general, the parallel field $\vec{B}_{\|}$can be oriented anywhere in the plane, which modifies $\alpha$ accordingly in the case that spin-orbit coupling is strong. Additionally, loosening the constraint that $\vec{B}_{\|}$lie parallel to $x$ introduces an artifact wavevector $q_{y} \approx 2 \pi B_{\|} \sin (\beta) d / \Phi_{0}$, where $d$ is the height difference between the centers of the quantum well and of the superconducting leads, and $\beta$ is the angle between the parallel magnetic field and the $x$ axis. This additional phase arises due to the magnetic flux penetrating the area $d L$ formed between the leads and the quantum well due to this height difference. Importantly, no flux penetrates this area when the parallel component of magnetic field is only in the $x$ direction, so that in this case the pair momentum is solely determined by the Zeeman coupling and the spin-orbit coupling.

The behavior of Josephson interference in our devices essentially involves different mechanisms when the parallel magnetic field lies in the $x$ or $y$ direction. With the above modeling it is clear that this difference is due to the dependence of $q_{y}$ on the magnetic field angle $\beta$, so that the data for the magnetic 
field $B_{y}$ reflects primarily the difference in height between the superconducting leads and the quantum well. Since we cannot entirely rule out either dominant Zeeman coupling or dominant SIA, we model both possibilities for this field direction. With dominant SIA and the height difference $d$ set to either $10 \mathrm{~nm}$ (Supplementary Figure 8a) or $70 \mathrm{~nm}$ (Supplementary Figure 8b), the model agrees well with the experimental observation. The corresponding model without any spin-orbit coupling also agrees, however (Supplementary Figure 8c, d).

Nevertheless, it is clear that with the parallel magnetic field in the $y$ direction, the most prominent feature in the response of the device is driven by the parallel magnetic flux penetrating the area $d L$ and not by effects intrinsic to the heterostructure. Assuming that only this parallel magnetic flux contributes, one can estimate the distance $d$ for each device, accounting for the slight difference in $W_{S C}$ for aluminum and niobium devices ( $1 \mu \mathrm{m}$ and $400 \mathrm{~nm}$ respectively). For devices A-E, the corresponding distance $d \approx 21,10,9,7$, and $70 \mathrm{~nm}$, in agreement with lithographic dimensions. The similar values of $d$ for devices B-D reflects the fact that these devices were all fabricated concurrently. Device E, in which niobium was used for the leads, has a much larger value of $d$ due to the fact that the niobium thickness was larger than the aluminum thicknesses.

Athough the parallel magnetic flux dominates the response of devices to the field $B_{y}$, with purely SIA it is still in principle possible in this direction to extract the intrinsic nature of spin-orbit coupling. Since the wavevectors $q_{y}$ and $\Delta k$ add and subtract, the ' $\mathrm{V}$ ' shape of supercurrent evolution contains two nearly identical slopes, which in our measurements are unobservable due to the concurrent decay of superconductivity. However, normalizing the theoretical critical current magnitude still reveals the possibility to determine the nature of spin-orbit coupling using this parallel field direction (Supplementary Figure 8e).

An additional characteristic common among the data sets is an asymmetry in the interference pattern upon inversion of one component of the applied magnetic field. In Supplementary Figure $8 \mathrm{f}$ we show an interference pattern measured on Device $\mathrm{F}$ with both positive and negative components $B_{y}$ and $B_{z}$. Here the data appears invariant under inversion of both components of the magnetic field, as we expect from time-reversal symmetry. However, the lack of symmetry under inversion of a single component of the magnetic field suggests that devices lack structural symmetry under rotation by 180 degrees. We may model this asymmetry as arising from a difference in lengths of the two leads on either side of the junction. An exaggeration of this effect, where the interface to one lead is 4 microns and to the other is 4.5 microns, shows increased intensity of interference for positive perpendicular field as compared to negative perpendicular field (Supplementary Figure 8g), similar to what is observed experimentally.

Finally, in all of the above modeling we have ignored contributions due to reflections at the ends of 
the mesa. In Supplementary Figure 8h, we plot the expected evolution of interference upon increasing $B_{y}$ assuming dominant SIA and with $d=0$, ignoring the possibility of reflections at mesa boundaries. We may include specular reflections at the mesa boundaries in the model, as discussed in Supplement X. With these contributions, the interference evolution is quantitatively modified (Supplementary Figure $8 \mathrm{i}$ ). However, the ' $\mathrm{V}$ ' shape of the interference evolution is still present, with each arm of the ' $\mathrm{V}$ ' having the same slope as was obtained by ignoring edge reflections. Hence, we conclude that the contribution of specular reflections preserving the spin direction only quantitatively modifies the expected device behavior. We have not carried out calculations for other boundary conditions, such as diffuse reflection, but we expect that results in these cases would not be qualitatively different from the cases of specular reflection or no reflection at all. 


\begin{tabular}{ccccccccccc}
$A(\mathrm{eV} \cdot \AA)$ & $B\left(\mathrm{eV} \cdot \AA^{2}\right)$ & $D\left(\mathrm{eV} \cdot \AA^{2}\right)$ & $M(\mathrm{eV})$ & $h(\mathrm{eV})$ & $\theta^{t h}$ & $R_{0} /(e \mathcal{E})\left(\mathrm{nm}^{2}\right)$ & $\mathcal{E}(\mathrm{mV} / \mathrm{nm})$ & $g_{E \perp}$ & $g_{H \perp}$ & $g_{\|}$ \\
\hline 3.645 & -68.6 & -51.2 & -0.010 & $0,0.0016$ & 0 & -15.6 & 0,10 & 22.7 & -1.21 & -20.5
\end{tabular}

Table 1: List of parameters used to model the band structure. Parameters correspond to a quantum well in the inverted regime, with a well width of $70 \AA$.

\section{Wafer I}

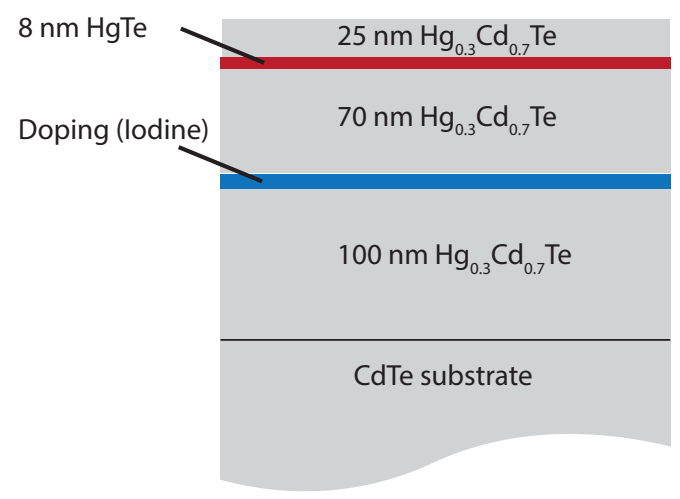

Wafer II

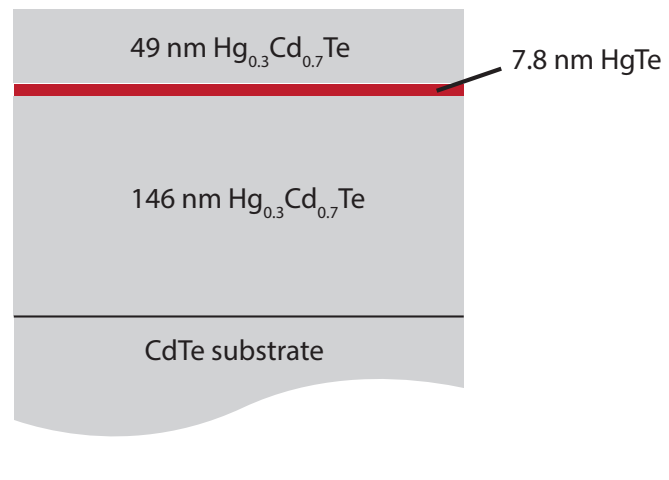

Supplementary Figure 1: Composition of the heterostructures used in the experiment, labeled Wafer I and Wafer II. Both wafers consist of a $\mathrm{HgTe}$ quantum well surrounded by barriers of $\mathrm{Hg}_{0.3} \mathrm{Cd}_{0.7} \mathrm{Te}$. In Wafer I, a layer containing iodine dopants lies $70 \mathrm{~nm}$ below the quantum well. Both wafers were grown on CdTe substrates, in the [001] crystal direction.

a.

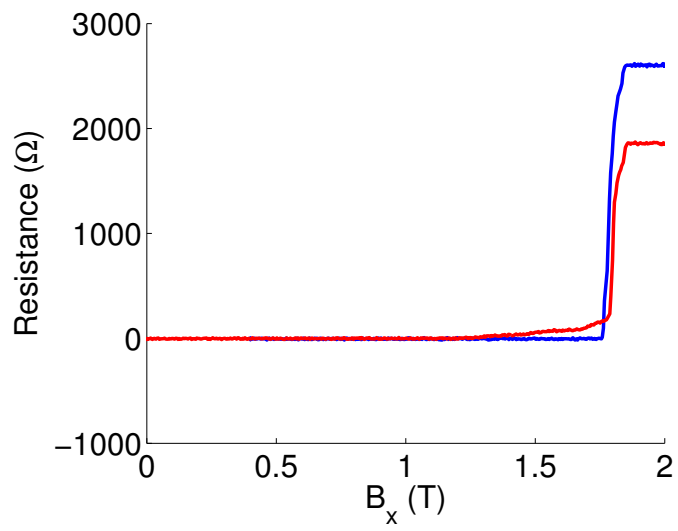

b.

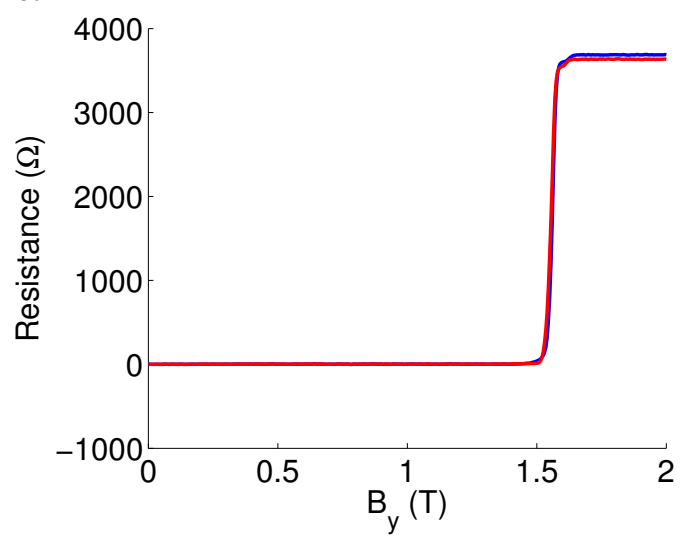

Supplementary Figure 2: Resistances of junction leads as the parallel magnetic field is increased in the $x$ and $y$ directions, for junctions with aluminum leads. The critical field in the plane of the leads is consistently above $1.5 \mathrm{~T}$. 


\section{a. Critical Current}

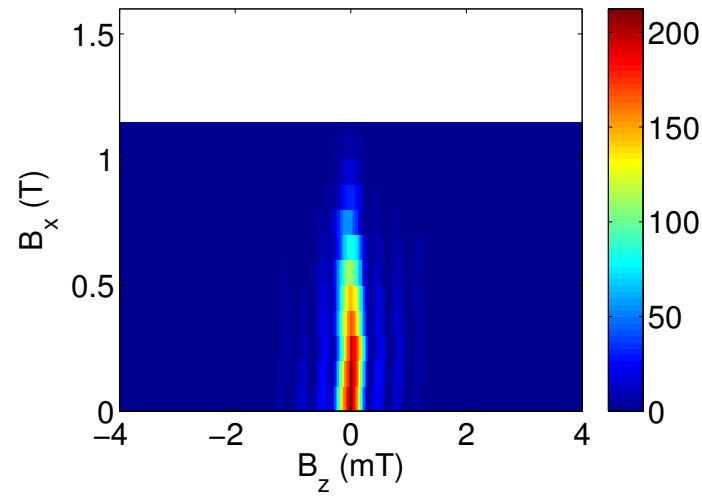

c. Normalized Critical Current

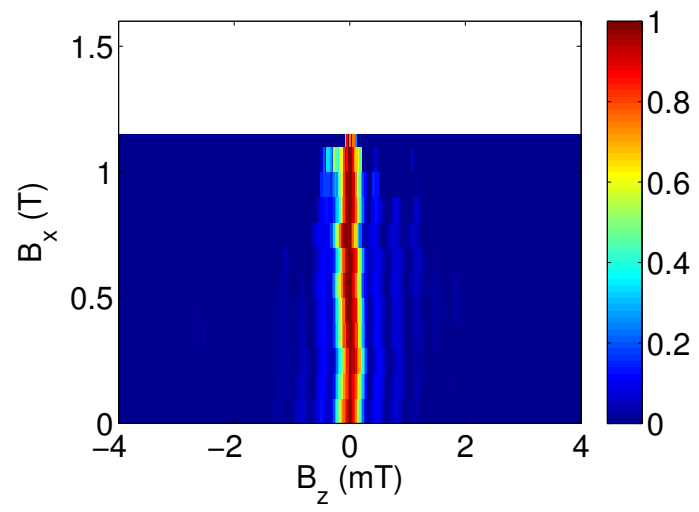

\section{b. Critical Current}

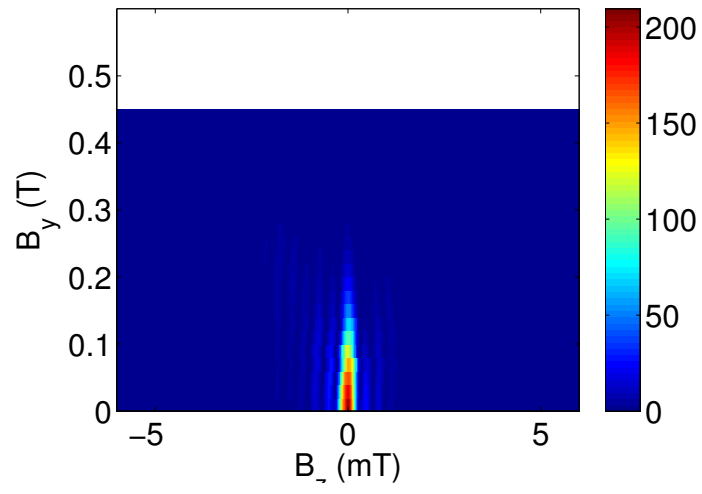

d. Normalized Critical Current

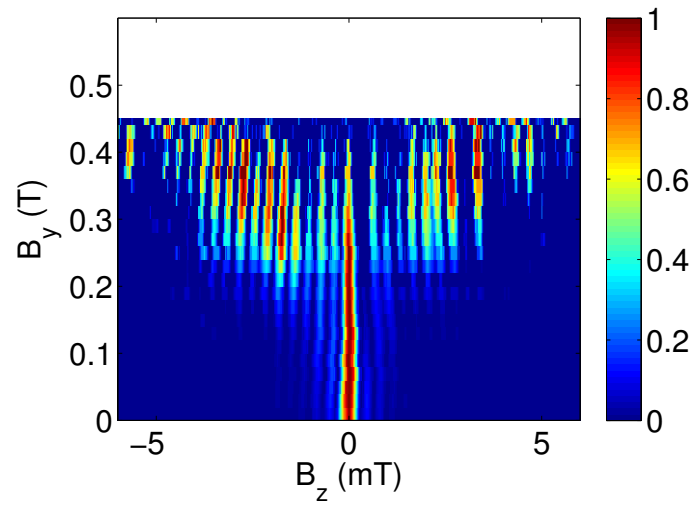

Supplementary Figure 3: The critical current as a function of perpendicular magnetic field $B_{z}$, as parallel components of the magnetic field are varied. The data presented here was taken using the device with aluminum leads presented in the main text (device D). a, b) As the parallel magnetic field in either the $x$ or $y$ direction is increased, the magnitude of the maximum critical current decreases. This decrease occurs more rapidly in $y$ direction than in the $x$ direction. c) Normalizing the Fraunhofer interference at each value of $B_{x}$ shows that the shape of the interference pattern remains essentially unaffected until it becomes immeasurably small. d) In the $B_{y}$ direction, normalization reveals a dramatically different behavior of the Fraunhofer interference, where critical current maxima occur at higher values of $B_{z}$ as $B_{y}$ is increased. Concurrently, the weight of the critical current at $B_{z}=0 \mathrm{mT}$ decreases to 0 . These observations match those deduced through measurements of the differential resistance as the parallel magnetic field varies in either the $x$ or $y$ direction. Therefore both differential resistance and critical current measurements reflect the same basic phenomenon. 
a.

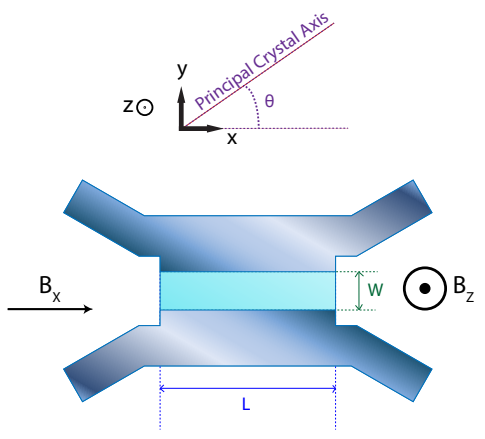

c. Device B: Aluminum, $\boldsymbol{\theta}=\mathbf{0}$

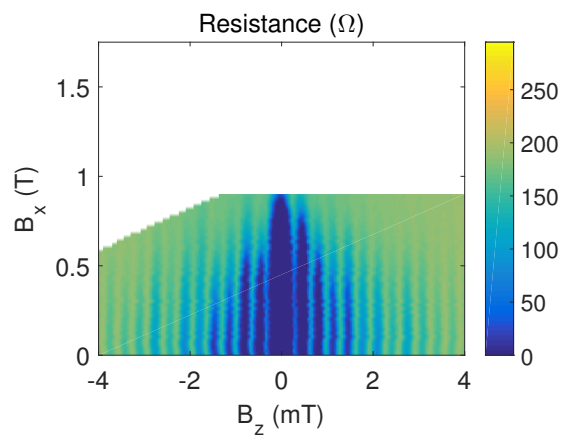

e. Device D: Aluminum, $\theta=\pi / 4$

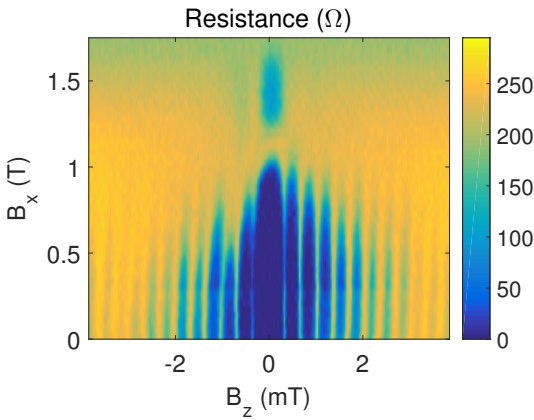

b. Device A: Aluminum, $\theta=\pi / 4$

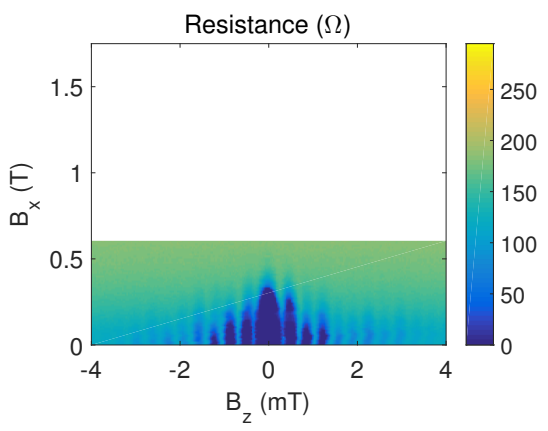

d. Device C: Aluminum, $\boldsymbol{\theta}=\pi / 2$

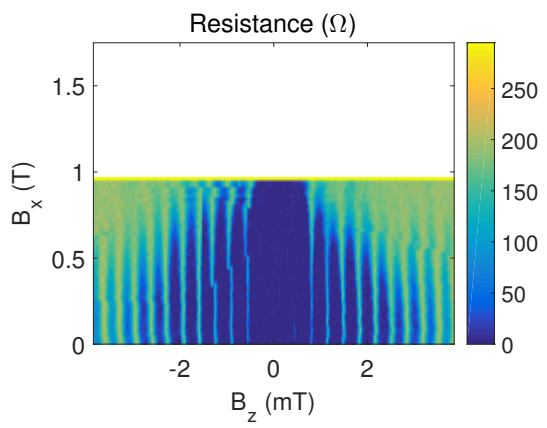

f. Device E: Niobium, $\theta=\pi / 4$

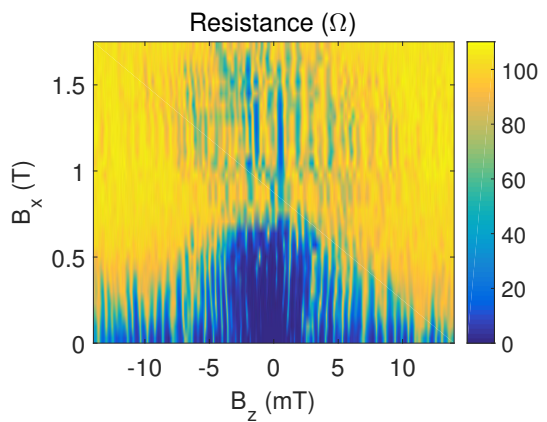

Supplementary Figure 4: Josephson interference as the magnetic field $B_{x}$ is increased. a) Junctions were oriented at an angle $\theta$, modulo $\pi / 2$, with respect to the [100] axis of the crystal. Devices generating data in (c-e) were concurrently fabricated with respect to the same crystal cleavage edge. Devices in (b) and (f) were separately fabricated. All junctions had aluminum leads except for in (f), where niobium leads were used. b) For a junction oriented at $\theta=\pi / 4$ with respect to the crystal, the differential resistance is monitored as both the perpendicular field $B_{z}$ and the parallel field $B_{x}$ are altered. As $B_{x}$ increases, the position of nodes in the interference pattern does not change, but the interference gradually disappears. c) A junction oriented at $\theta=0$ and $\mathbf{d}$ ) a junction oriented at $\theta=\pi / 2$ with respect to the crystal show qualitatively identical behavior. e) A further junction aligned at $\theta=\pi / 4$ shows the same behavior, as previously presented in the main text. f) Also in the main text, the junction with niobium leads is oriented with $\theta=\pi / 4$ and shows interference which remains strongly weighted at $B_{z}=0 \mathrm{~T}$. The observations on aluminum devices are all consistent with dominant SIA in the quantum well. 
a.

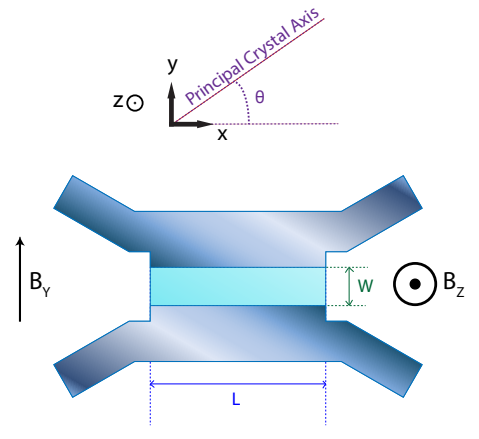

c. Device B: Aluminum, $\boldsymbol{\theta}=\mathbf{0}$

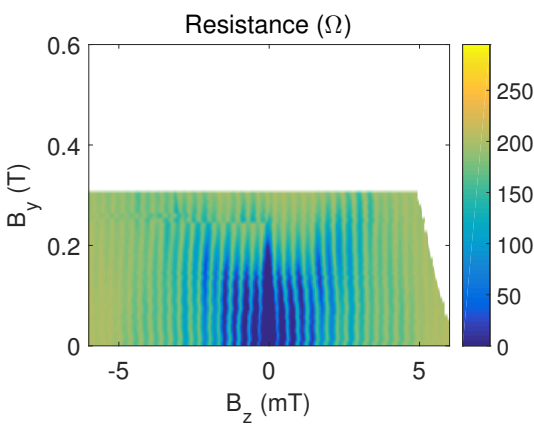

e. Device $D$ : Aluminum, $\theta=\pi / 4$

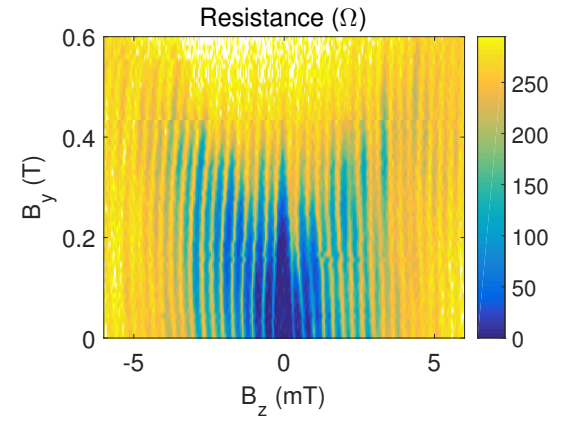

b. Device A: Aluminum, $\theta=\pi / 4$

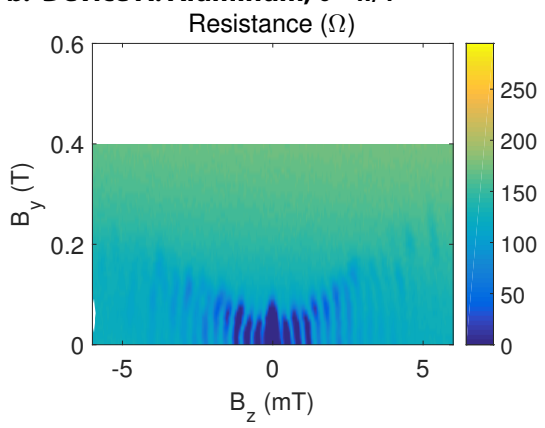

d. Device C: Aluminum, $\boldsymbol{\theta}=\pi / 2$

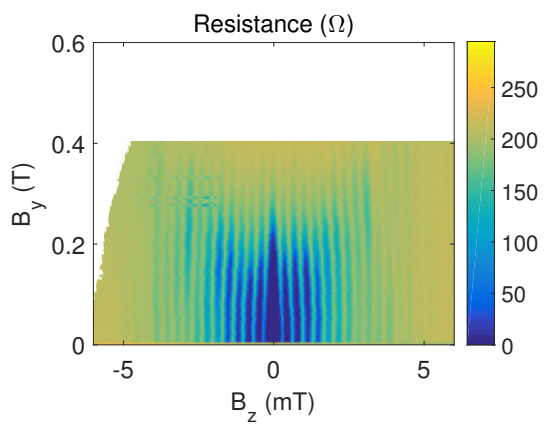

f. Device E: Niobium, $\theta=\pi / 4$

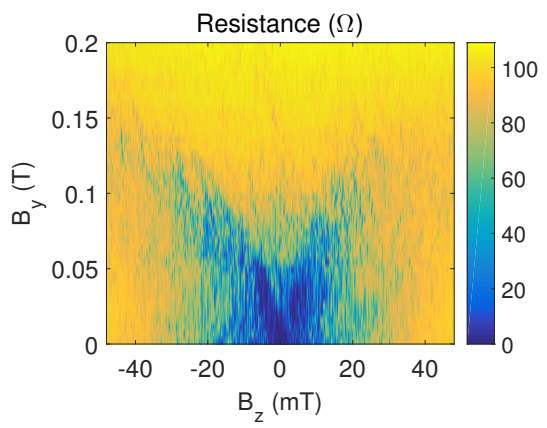

Supplementary Figure 5: Josephson interference as the magnetic field $B_{y}$ is increased. a) Junctions were oriented at an angle $\theta$, modulo $\pi / 2$, with respect to the [100] axis of the crystal. As in Supplementary Figure 4, devices generating data in (c-e) were concurrently fabricated with respect to the same crystal cleavage edge, and devices in (b) and (f) were separately fabricated. b) With the junction aligned such that $\theta=\pi / 4$, the differential resistance is monitored as a function of the perpendicular field $B_{z}$ and the parallel field $B_{y}$. Increasing $B_{y}$ rapidly causes the weight of interference fringes to shift to larger $B_{z}$ values, forming a ' $\mathrm{V}$ ' shape. The interference evolves more rapidly due to a parallel field in the $y$ direction than in the $x$ direction due to the fact that leads are spatially displaced in $z$ with respect to the quantum well. c) Orienting a junction at $\theta=0$ introduces no qualitative change to the behavior, as is also the case with $\mathbf{d}$ ) a junction oriented at $\theta=\pi / 2$. Junctions from the main text with e) aluminum and f) niobium leads are presented, also displaying similar behavior. The enhanced scale of $B_{z}$ in the niobium-based junction is due to the increased thickness of the leads. 
a.

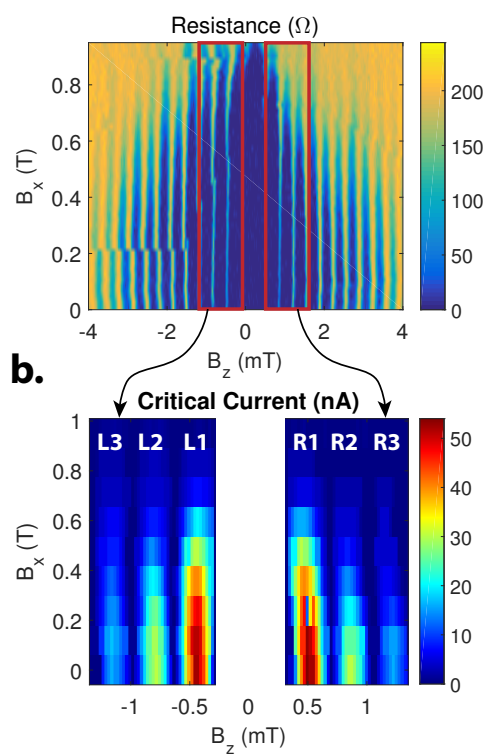

c.

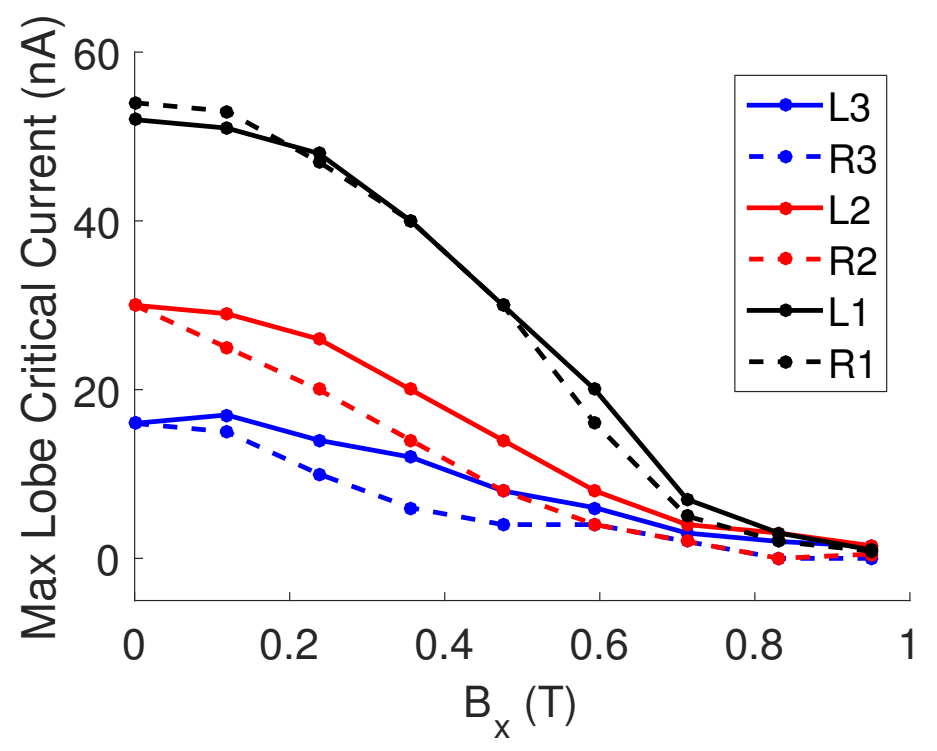

Supplementary Figure 6: Evolution of critical currents, in Device C, oriented such that $\theta=\pi / 2$. Data was taken with the top gate voltage set to $0 \mathrm{~V}$. a) The differential junction resistance, measured with no applied DC current, evolves consistently with the absence of BIA in the device. b) The critical currents in the interference side lobes adjacent to the central lobe decay as the parallel field $B_{x}$ increases from zero. The region of the critical current measurement is outlined in red in (a). Each side lobe is labeled in white. c) The extracted maximum critical current for each side lobe. Within each lobe, the critical current decreases from its maximum value at $B_{x}=0 \mathrm{~T}$. 


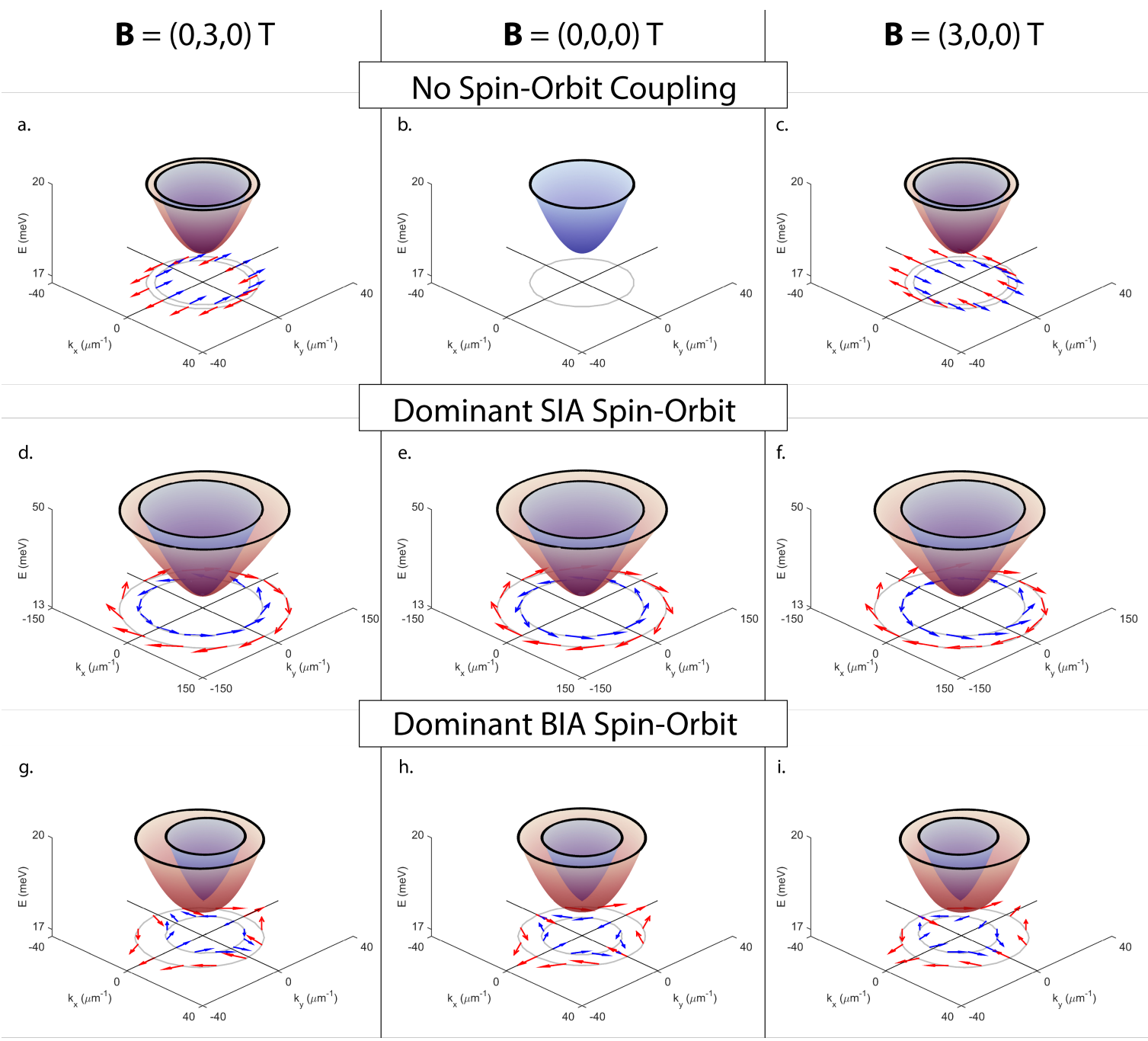

Supplementary Figure 7: Modeling of the conduction band structure under various spin-orbit and parallel magnetic field conditions. a-c) When spin-orbit coupling is absent, the addition of a $3 \mathrm{~T}$ parallel magnetic field simply polarizes spins in a direction determined by the sign of the in-plane g-factor, $g_{\|}$. With no exernal magnetic field, the two spin bands are degenerate (b). d-f) Dominant structural inversion asymmetry causes axially symmetric spin-splitting to occur at finite momentum (e). This type of spin-orbit coupling causes the bands to shift orthogonally to the external magnetic field $(\mathrm{d}, \mathrm{f})$, leading to nonzero Cooper pair momentum in the shift direction. g-i) Dominant bulk inversion asymmetry with $\theta^{t h}=0, \pi / 2$ acts oppositely to structural inversion asymmetry, so that external magnetic fields cause bands to shift parallel to the external magnetic field. This type of shift is not observed experimentally in our devices. 


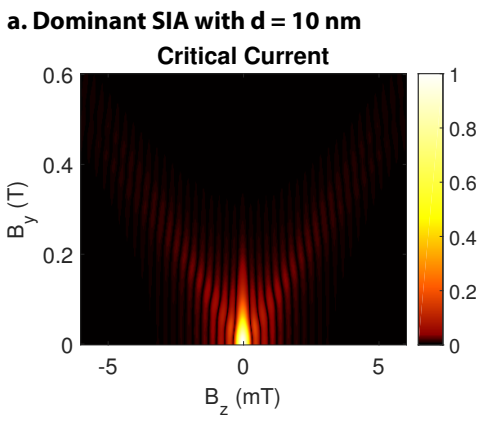

d. Dominant Zeeman with $\mathbf{d}=70 \mathrm{~nm}$

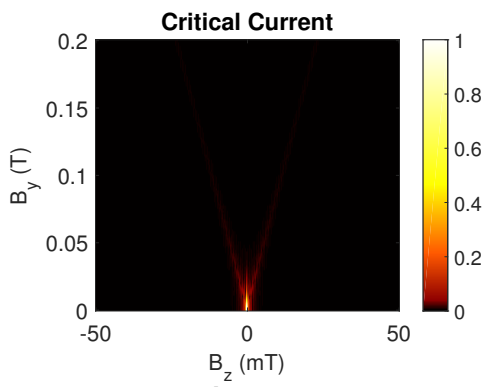

g. Dominant SIA, $d=10 \mathrm{~nm}$, asymmetry

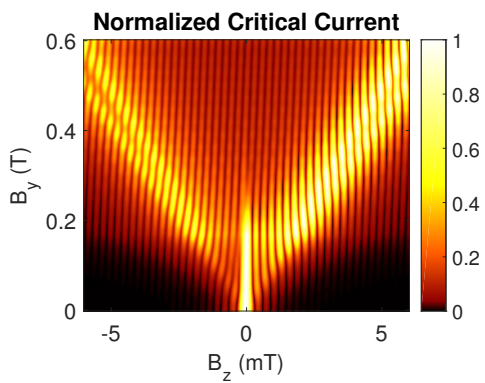

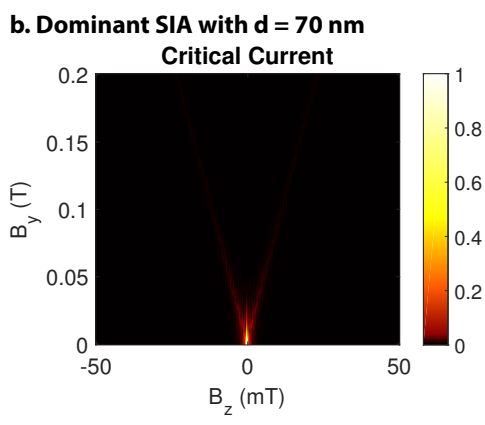

e. Dominant SIA with $\mathrm{d}=10 \mathrm{~nm}$

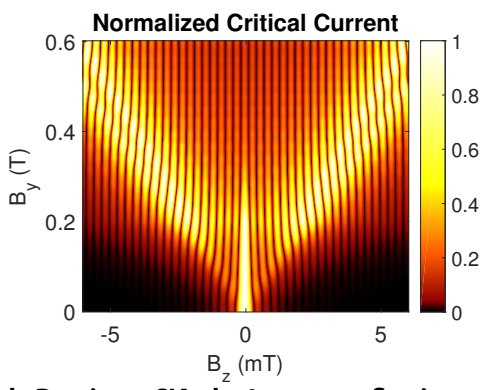

h. Dominant SIA, $d=0 \mathrm{~nm}$, no reflections

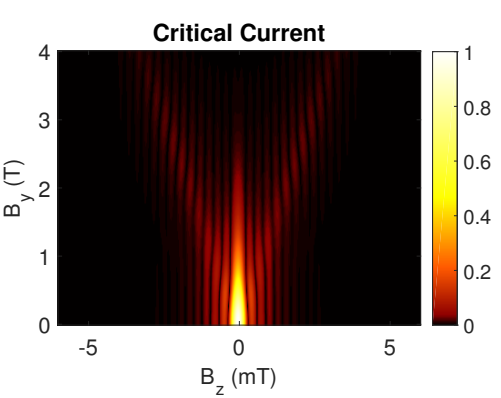

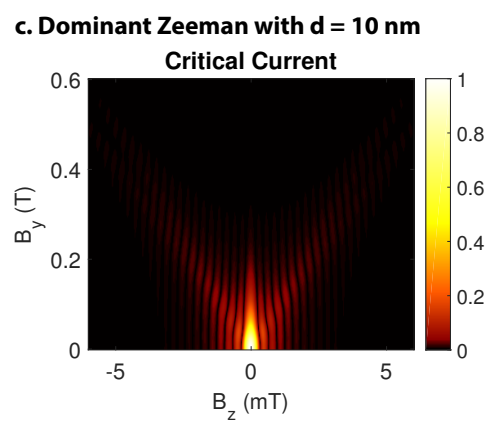

f. Resistance Measured with $\mathrm{B}_{\mathrm{y}}>\mathbf{0}$ and $\mathrm{B}_{\mathrm{y}}<0$

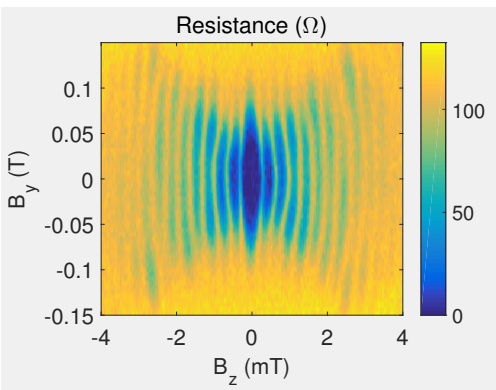

i. Dominant SIA, $d=0 \mathrm{~nm}$, reflections Critical Current

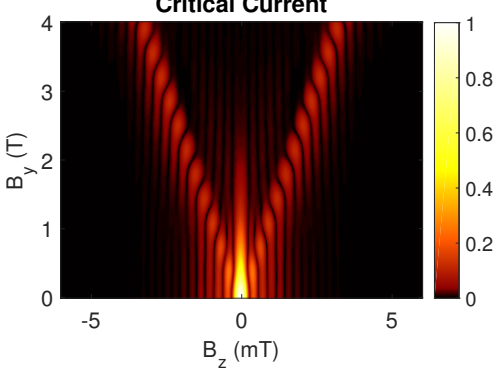

Supplementary Figure 8: Modeling of the critical current as the perpendicular magnetic field $B_{z}$ (generating flux quanta) and the parallel magnetic field $B_{y}$ (generating Cooper pair momentum) are varied. a) With dominant SIA and the height difference $d$ between the leads and the quantum well set to $10 \mathrm{~nm}$, the interference evolves consistently with measurements of differential resistance on aluminum devices (devices A-D). b) Increasing $d$ to $70 \mathrm{~nm}$ decreases the slope of each arm of the interference pattern consistently with the measurement of a device with thickner niobium leads (device E). c, d) Eliminating spin-orbit coupling leads to a similar picture for both values of $d$, highlighting the overwhelming extrinsic nature of the pairing momentum induced when the parallel field is applied in the $y$ direction. e) Normalizing the critical current at each value of $B_{y}$ reveals additional features weakly present in the interference with SIA and $d=10 \mathrm{~nm}$. Since the extrinsic wavevector $q_{y}$ adds and subtracts with the wavevector $\Delta k$ induced due to SIA, two slopes are in principle found in each arm of the interference pattern. However, superconductivity weakens to the extent that such splitting cannot be conclusively observed in our devices. f) The resistance of Device F, measured as both $B_{y}$ and $B_{z}$ are tuned to positive and negative values. The measured resistance is observed to be symmetric under inversion of both $B_{y}$ and $B_{z}$, as expected from time-reversal symmetry. Under inversion of either $B_{y}$ or $B_{z}$, however, the resistance is asymmetric. g) Modeling asymmetry in the lengths of superconducting electrodes leads to asymmetry in the interference with respect to inversion of $B_{z}$. Plotted here are the expected critical currents for a device with 4 microns and 4.5 microns as the interfacial lengths. h) The expected evolution of interference upon increasing $B_{y}$ assuming dominant SIA and with $d=0$. i) Including specular reflections at the mesa ends, assuming the presence of a steep confining potential which does not flip spins upon reflection, quantitatively modifies the interference evolution. However, qualitatively the behavior remains unchanged. 